Revue musicale OICRM

\title{
Matériaux anciens dans la musique contemporaine actuelle. Postmodernisme et modernisme en questions
}

\section{Liouba Bouscant, Michel Gonneville et Ofer Pelz}

Volume 2, numéro 2, 2015

Une relève

URI : https://id.erudit.org/iderudit/1060129ar

DOI : https://doi.org/10.7202/1060129ar

Aller au sommaire du numéro

Éditeur(s)

OICRM

ISSN

2368-7061 (numérique)

Découvrir la revue

Citer cet article

Bouscant, L., Gonneville, M. \& Pelz, O. (2015). Matériaux anciens dans la musique contemporaine actuelle. Postmodernisme et modernisme en questions. Revue musicale OICRM, 2(2), 1-43. https://doi.org/10.7202/1060129ar
Résumé de l'article

Cet article vise à contribuer à la réflexion sur le procédé de l'emprunt thématique dans la composition au XXIe siècle, en étudiant plus particulièrement le cas de deux compositeurs actuels reconnus, établis au Canada : Michel Gonneville né en 1950 et Ofer Pelz né en 1978. Il s'agit de comprendre, après des décennies de structuralisme et de postmodernisme, le sens et l'apport de l'alliance entre le langage contemporain du début des années 2000 et des matériaux thématiques passés issus de la musique savante. Peut-on analyser celle-ci encore comme postmoderne et, si tel n'est pas le cas, quelle alternative apparaît ? 


\title{
Matériaux anciens dans la musique contemporaine actuelle. Postmodernisme et modernisme en questions
}

\section{Liouba Bouscant}

\author{
avec la collaboration de \\ Michel Gonneville \\ et Ofer Pelz
}

\begin{abstract}
Résumé
Cet article vise à contribuer à la réflexion sur le procédé de l'emprunt thématique dans la composition au $\mathrm{XXI}^{\mathrm{e}}$ siècle, en étudiant plus particulièrement le cas de deux compositeurs actuels reconnus, établis au Canada : Michel Gonneville, né en 1950, et Ofer Pelz, né en 1978. Il s'agit de comprendre, après des décennies de structuralisme et de postmodernisme, le sens et l'apport de l'alliance entre le langage contemporain du début des années 2000 et des matériaux thématiques passés issus de la musique savante. Peut-on analyser celle-ci encore comme postmoderne et, si tel n'est pas le cas, quelle alternative apparaît?
\end{abstract}

Mots clés : emprunt; modernisme; musique contemporaine; postmodernisme; $\mathrm{XXI}^{\mathrm{e}}$ siècle.

\section{Abstract}

This article reflects on thematic quotation from past art-music in contemporary composition, after decades of structuralism and postmodernism, by studying particularly two known composers established in Canada: Michel Gonneville, born in 1950, and Ofer Pelz, born in 1978. Could we still analyse these compositional styles as postmodernism and, if this is not the case anymore, which alternative comes up?

Keywords: borrowing; contemporary music; modernism; postmodernism; 21st century. 
DÉPASSER L'ANTITHÈSE POSTMODERNISME VERSUS MODERNISME

Nous nous proposons de contribuer à la réflexion sur le procédé de l'emprunt de matériaux appartenant au répertoire savant passé dans la composition au début $\mathrm{du} \mathrm{xxI}^{\mathrm{e}}$ siècle, dans le contexte actuel du dépassement du postmodernisme et de la reconnaissance d'un modernisme en prolongement. Nous étudierons, à cette fin, le cas de deux compositeurs actuels aux démarches opposées : celui du canadien Michel Gonneville, né en 1950, qui a été notamment élève de Gilles Tremblay, Karlheinz Stockhausen et Henri Pousseur, et celui d'Ofer Pelz, né en 1978, appartenant à la jeune génération de compositeurs récompensés par de nombreux prix internationaux, et notamment invité à la Biennale de Venise à l'automne 2014.

L'utilisation de matériaux préformés empruntés au passé appartient à la tradition musicale savante dès le Moyen Âge. Outre le procédé de composition polyphonique à partir d'un cantus firmus fixé dans le répertoire du chant grégorien, sont ensuite apparus, dès l'aube de la Renaissance, les "déploration ", " tombeau " ainsi que diverses formes de commémoration des maîtres défunts ou de leurs œuvres, utilisant notamment les procédés de la citation et de l'acrostiche, devenant des genres établis, appartenant aux catégories du défi compositionnel, de la revendication d'un héritage ou encore del'hommage. Même les modernistes les plus ardents de la période précédant Darmstadt ont intégré des matériaux passés dans certaines de leurs compositions : Stravinsky, par exemple, dans son Momemtum pro Gesualdo en 1960, "recompose " un hommage stylistique fragmentant le madrigal de Gesualdo en groupes opposés avec divers timbres et tonalités, selon des principes d'enchaînements harmoniques non authentiques. Schoenberg, en réinvestissant l'écriture haendélienne et du concerto grosso baroque dans son Concerto pour quatuor à cordes et orchestre (1933) d'après le Concerto grosso op. $6 \mathrm{n}^{\circ} 7$ de Haendel, construit une vision téléologique de l'évolution du langage musical, dont il se pose comme le médiateur. Il s'agit cependant de comprendre, après des décennies de postmodernisme dominant, les motivations qui poussent les compositeurs au début du XXI siècle à opérer l'alliance entre un langage contemporain et des matériaux thématiques passés issus de la musique savante. L'enjeu prégnant est, plus largement, celui de la réception de l'héritage musical occidental par les compositeurs actuels de musique contemporaine, du rapport de ces derniers à l'histoire et de leur attitude face au passé, à la contemporanéité et à l'avenir.

Le postmodernisme musical désigne une période de l'histoire dont l'émergence a lieu dès la fin des années 1960, succédant au modernisme de l'École de Darmstadt ${ }^{1}$. Insaisissable dans une globalité conceptuelle homogène, le postmodernisme a régi la création sans laisser aux compositeurs le choix de lui obéir ou non et sans se laisser définir de façon univoque (Harvey 1990). Nombreux sont les compositeurs refusant pourtant de se laisser revêtir de l'étiquette « postmodernistes ». Certes, l'attitude postmoderniste semble à première vue reposer sur la même logique que le modernisme

1 David Harvey, quant à lui, entrevoit le tournant postmoderniste dans l'année 1972 (Harvey 1990, p. vii). 
dont elle condamne justement l'idéologie de la rupture, puisqu'elle en prend le " contrepied ", comme le fait remarquer Guy Scarpetta en 1985 dans L'impureté (p. 16). Ce dernier pose la question : « ...peut-on échapper à l'idéologie avant-gardiste sans pour autant congédier tout l'apport de la modernité littéraire et artistique du siècle ? [...] Comment s'affranchir de l'esthétique de la radicalité tout en préservant les valeurs de l'invention? » (ibid., p. 19). Il s'est agi en réalité, pour le postmodernisme, déclare Scarpetta, de "sortir de l'ère des ruptures " (ibid., p. 28). En effet, Jameson définit le postmodernisme non comme un style homogène, mais comme une vaste période de l'histoire et comme une culture dominante (Jameson 1984, p. 55). Les facteurs de l'émergence de ce courant sont analysés de façons variées et selon une tendance souvent marxiste : 1'ère du capitalisme parvenu à un stade avancé (ibid.) les transformations économiques, la suraccumulation capitaliste et les progrès technologiques qui instaurent une façon compressée, inédite, d'expérimenter l'espace et le temps, entraînant la primauté de l'image sur le récit, de l'éphémère et du fragmentaire sur le durable, de l'esthétique sur l'éthique (Harvey 1990), les nouveaux flux et modes informatisés et démultipliés d'acquisition du savoir modifiant les modes de transmission et de formation de celui-ci (Lyotard 1979). De notre côté, nous référant en partie à Jean-François Lyotard, il nous semble possible de définir le postmodernisme comme une perte de foi, une incrédulité face aux idéologies politiques ou du progrès, un vaste scepticisme général à l'égard de la raison positiviste et universaliste. Nous nous appuyons sur Lyotard et son critère de métarécits - grandes idéologies ou philosophies régissant les activités et les idéaux modernes (telles le récit chrétien, le récit Aufklärer, le récit spéculatif, le récit capitaliste, le récit marxiste) - qui évoluent en micro-narrativités disséminées dans le champ de la création contemporaine et se logeant à l'échelle des esthétiques individuelles ou des écoles (ibid., p. 7 et p. 36). La désillusion à l'égard du mythe du progrès et du positivisme explique les microrécits, la diversité ainsi que l'éclectisme des styles et des choix esthétiques. S'observe alors une coexistence de styles présentant des traits postmodernes avec des styles à tendance moderniste comme le résume Kenneth Gloag selon lequel elle ne fait qu' « intensifier la pluralité et la diversité du spectre culturel : la musique moderniste comme option stylistique dans le contexte d'un monde postmoderne »(Gloag 2012, p. 164)2.

Ces aspects modernisant et postmoderniste au sein de l'époque éponyme englobante se décèlent dans l'attitude face au passé. Chaque œuvre devient en effet une réponse particulière et individuelle à la question primordiale du rapport à l'histoire et à la tradition. Le postmodernisme en musique est caractérisable par un faisceau d'attitudes face à l'histoire et au patrimoine musical opposées à celles du modernisme, mises en lumière par de nombreux théoriciens et musicologues. Le tableau ci-dessous offre un essai de synthèse de la confrontation entre modernisme et postmodernisme tel qu'il a pu être observé au cours des années 1970, 1980 et 1990.

2 «[T] he presence of [...] music [that]can be identified with modernism continues to be produced [...] only intensified the plurality and diversity of the cultural spectrum: modernist music as a stylistic option in a postmodern context »; notre traduction. 


\begin{tabular}{|c|c|}
\hline MODERNISME & POSTMODERNISME \\
\hline Evolutionnisme & Fin de l'histoire \\
\hline Progressisme & $\begin{array}{l}\text { Dénégation du mythe du progrès et du } \\
\text { dépassement }\end{array}$ \\
\hline Complexité & Simplicité \\
\hline $\begin{array}{l}\text { Mouvement tourné vers l'avenir } \\
\text { (Rea 1997, p.61) }\end{array}$ & $\begin{array}{l}\text { Mouvement tourné vers le passé } \\
\text { (Rea 1997, p. 61) }\end{array}$ \\
\hline $\begin{array}{l}\text { Culte de la nouveauté (" néopathie », } \\
\text { Nattiez 1993, p. 161), } \\
\text { Dialectique de l'ancrage dans la tradition } \\
\text { versus radicalisme }\end{array}$ & $\begin{array}{l}\text { Fin des clivages nouveauté/passé; invention/ } \\
\text { académisme, rupture/continuité entre } \\
\text { révolution/réaction }\end{array}$ \\
\hline $\begin{array}{l}\text { Peut faire « table rase », avant-garde (ex. } \\
\text { ready-made) comme référence }\end{array}$ & $\begin{array}{l}\text { Sortie de 1' «ère des ruptures" } \\
\text { (Scarpetta 1985, p. 28) }\end{array}$ \\
\hline $\begin{array}{l}\text { Authenticité du discours, premier degré, } \\
\text { référencialité immédiate }\end{array}$ & $\begin{array}{l}\text { Toute l'œuvre est « métalangage », " second } \\
\text { degré » (Scarpetta 1985, p. 29). "Mise en } \\
\text { scène » de la «fascination » à l'égard du passé } \\
\text { (Ramaut-Chevassus 1998, p. 104). Le passé } \\
\text { utilisé avec «artifice » (Scarpetta 1985) }\end{array}$ \\
\hline $\begin{array}{l}\text { Universalisme } \\
\text { Autonomie de l'œuvre d'art }\end{array}$ & $\begin{array}{l}\text { Esthétique de l' «impureté »(Scarpetta 1979). } \\
\text { Acceptation de la diversité, de la pluralité, } \\
\text { des cultures multiples. La musique n'est plus } \\
\text { autonome, mais soumise à des contextes } \\
\text { sociaux, politiques, culturels (Kramer 2002, } \\
\text { p. 16) }\end{array}$ \\
\hline $\begin{array}{l}\text { Musique poḯtico-centrique } \\
\text { (Nattiez 1993, p. 180) }\end{array}$ & $\begin{array}{l}\text { Musique esthésico-centrique } \\
\text { (Nattiez 1993, p. 180) }\end{array}$ \\
\hline Esthétique de la spécificité des codes & $\begin{array}{l}\text { Défie les barrières entre haut-style et bas-styles, } \\
\text { entre valeurs élitistes et valeurs populaires } \\
\text { (Kramer } 2002, \text { p. } 16 \text { ) }\end{array}$ \\
\hline $\begin{array}{l}\text { Culte de la forme, « synthèse », } \\
\text { "structure », hiérarchisation, « design » } \\
\text { formel (Hassan 1991, p. 91) }\end{array}$ & $\begin{array}{l}\text { "Anarchie », « hasard "(Hassan 1991, p. 91); } \\
\text { Forme, processus }\end{array}$ \\
\hline $\begin{array}{l}\text { Transformation du matériau source, } \\
\text { citations exceptionnelles, dans un contexte } \\
\text { sémantique précis }\end{array}$ & Emprunt, citation, collage \\
\hline Unité, homogénéité & Eclectisme \\
\hline
\end{tabular}


Peut-on prédire, après plus de cinq décennies de postmodernité, l'imminence d'une nouvelle ère, ou même en faire le constat ? Tout d'abord, il convient de corriger la fausse alternative historique entre courant moderniste et courant postmoderniste ainsi que la vision simpliste et erronée de leur étanchéité.

Jean-François Lyotard a inclus le postmodernisme dans le modernisme, le définissant, paradoxalement, comme l'état naissant de la modernité, elle-même «mode de pensée ${ }^{3}$. Le compositeur et musicologue John Rea, quant à lui, ose prédire le futur, en jouant, à la suite de Lyotard, la carte du faux paradoxe : il fait se succéder, à grande échelle dans l'histoire, postmodernisme puis modernisme (Rea 1997, p. 56). David Harvey, bien que distinguant les deux courants, tient à faire valoir leur socle commun, dans la mesure où le postmodernisme conserve la moitié des caractéristiques du modernisme, tel qu'il a été défini par Baudelaire : la prédilection pour l'éphémère, le fragment, le discontinu et le chaos (Harvey 1990, p. 44). Mais surtout, depuis les années 1990, la réflexion esthétique menée notamment par Marjorie Perloff, Björn Heile et David Metzer, stigmatise l'opposition binaire entre modernisme et postmodernisme, tant sur le plan chronologique que philosophique et démontre la résilience du modernisme depuis les années 1970 jusqu'au début du $\mathrm{XXI}^{\mathrm{e}}$ siècle. Le modernisme accuse, montrent ces auteurs, une rémanence adoptant divers visages au fil de l'histoire. Alors qu'il marque une partie bien délimitée du $\mathrm{xx}^{\mathrm{e}}$ siècle $-1 \mathrm{es}$ décennies 1900-1940 - loin d'être englouti par le courant postmoderne, il s'impose encore au début $\mathrm{du} \mathrm{XxI}^{\mathrm{e}}$ siècle. Björn Heile tient cependant à compléter de façon fondamentale la théorie de Marjorie Perloff assignant au postmodernisme la destinée de "momentum» (Perloff 2002, p. 1-2). En effet, il soutient également la survie du modernisme : «Le modernisme demeure vital. Il n'a pas été supplanté. Il en tire une richesse d'idéaux et de précédents et est alimenté par des impulsions continuelles. Muni de telles ressources, il a franchi le vingt-et-unième siècle " (Heile 2009, p. 1) ${ }^{4}$. Cependant, il établit que le phénomène du modernisme n'accomplit pas une trajectoire linéaire reliant deux périodes historiques éloignées de cent ans, mais se prolonge au contraire de façon continue et anamorphique :

L'histoire du modernisme en musique est davantage construite comme une trajectoire continue (cependant loin d'être linéaire), que le fossé de cent ans [entre deux modernismes] de Perloff ne le présuppose, même implicitement. En outre, le concept du modernisme qui est surtout développé ici, est sujet au changement historique et à une définition constante et n'est pas une

\footnotetext{
3 Jean-François Lyotard développe cette thèse dans de nombreux écrits, dont La condition postmoderne. Voir également Lyotard (1986, p. 29-30) : «Une œuvre ne peut devenir moderne que si elle a d'abord été postmoderne. Le postmodernisme ainsi entendu n'est pas le modernisme à sa fin, mais à l'état naissant, et cet état est constant».

4 "[...] Modernism [...] remains vital. It has not been supplanted. It drawns upon a wealth of ideals and precedents and is fueled by continuing impulses. With such ressources, it has crossed over into the twenty-first century"; notre traduction.
} 
entité monolithique; la dernière thèse qui soit avancée est celle d'un "néomodernisme » ou d'une « rétro-avant-garde » (Heile 2009, p. 3) ${ }^{5}$.

Björn Heile emprunte plus particulièrement au philosophe Habermas la thèse d'une modernité à l'état de projet constant, qu'il valide avec la notion de modernisme : «En ce sens, le modernisme s'édifie, non comme une période historique délimitée, mais ainsi qu'Habermas le fait avec la modernité, comme un "projet inachevé" " (ibid. $)^{6}$. David Metzer, quant à lui, s'emploie à démontrer que le courant spectral et celui de la nouvelle complexité à la fin $\mathrm{du} \mathrm{xx}^{\mathrm{e}}$ siècle sont des représentants du modernisme (Metzer 2009). En outre, le modernisme ne se réduit pas, comme tient à le faire constater Andrew Timm, à l'exercice d'un langage atonal (Timms 2009).

Non seulement l'antithèse et l'exclusion de l'une des notions par l'autre sont caduques, tant historiquement que sur le plan philosophique, mais il est évident que le phénomène du postmodernisme, s'il s'avère être une période encore actuellement vécue, ne permet pas à ceux qu'il englobe de s'en extraire pour poser in abstracto des principes fossoyeurs. En effet, le propre du postmodernisme est justement d'exclure toute tentative - éminemment moderniste - de dépassement dialectique de l'histoire et des grands courants esthético-culturels, pensé et voulu consciemment, comme l'énonce Gloag7. Dès lors, déclarer la permanence du postmodernisme tient lieu d'une attitude postmoderniste prenant position contre le modernisme. À l'inverse, déclarer que la prise de position postmoderniste comme alternative au modernisme est dépassée est une posture en réalité fondamentalement moderniste s'appuyant sur l'évolution dialectique de la pensée et de la théorisation esthétique et historique de la musique.

Peut-on sortir actuellement, dès lors, du postmodernisme? L'impasse réflexive anticipatrice n'est cependant pas, selon nous, le triste lot du théoricien, mais elle devra être contournée par une étude approfondie et extrêmement documentée des œuvres et des compositeurs. Nous proposons sur ce point de laisser ici la parole à John Croft, qui envisage la forte probabilité d'une nouvelle ère esthétique en latence succédant à celles qui ont caractérisé respectivement le prémodernisme, le modernisme et le postmodernisme. Les trois premières ères citées ci-après par Croft sont mises en lumière par Jean-François Lyotard, tandis que la dernière est abordée par Giorgio Agamben :

$5 \quad$ " [...][T] he history of modernism in music is constructed as a more continuous, albeit far from linar trajectory than the 100-year gap Perloff is at least implicitly presupposing. Furthermore, the concept of modernism that is mostly developed here is subject to historical change and constant definition, not a monolithic entity; the last thing that is advocated is "neo-modernism" or "retro-avant-garde" "; notre traduction.

6 "In this sense, modernism is [...] constructed not as a close historical period, but, as Habermas does with modernity, as an incomplete project »; notre traduction.

7 «L'interrogation : comment, ou à partir de quand, théoriser la culture et écouter de la musique, après le postmodernisme, et, comment à la fois conceptualiser et représenter ceci, pourrait bien nécessiter, plutôt que des proclamations faciles, de nouvelles théories et concepts qui soumettent le postmodernisme au type de réponse critique qui était jadis dirigé contre le modernisme »; notre traduction de cet extrait : "How or when, we begin to theorize culture, and hear music, after postmodernism, and how that might be both conceptualized and represented, may well require, rather than such easy proclamations, new theories and concepts that subject postmodernism to the kind of critical response that was once projected against modernism » (Gloag 1992, p. 161). 
l'esthétique de la musica ficta, au contenu divin transcendant, celle de la musica fingens, une musique intellectualisée et spéculative telle que représentée notamment par Schoenberg, et les esthétiques postmodernes de la novatio - ou encore musica figura ${ }^{8}$ purement ludique - et de la collection (aesthtetic of the collector) (Agamben 1999) ${ }^{9}$. Cette dernière, éclectique et aliénatrice est en tout point tragique, telle que l'explication d'Agamben le laisse transparaître :

Le château de la culture est désormais devenu un musée où, d'une part, la richesse du passé, dans laquelle l'homme ne peut se reconnaitre, est accumulée pour être dédiée à la jouissance esthétique de la communauté et, d'autre part, cette jouissance n'est possible que par l'aliénation qui la prive de sa signification immédiate et de sa capacité poétique à ouvrir son espace à l'action et à la connaissance » (ibid., p. 111, cité par Croft 2009, p. 33) ${ }^{10}$.

Or, cette esthétique nouvelle qu'appelle de ses vœux John Croft et qu'il entrevoit dans la musique de Sciarrino se définit comme celle qui forgera de nouveau un lien solide et critique entre l'art, l'environnement et la culture, entre le présent et le passé, et dans laquelle la présence de ce dernier ne sera plus simple signe et réification parodique vidée de contenu et d'aura, mais un matériau, un geste et une énergie diffus dans l'œuvre. En d'autres termes, il entrevoit la possible émergence d'une nouvelle sorte de mimesis.

Il ne nous appartient pas dans ce bref article de répondre à la question de l'actualité et de la pertinence, encore au début du Xxi siècle, de la notion de postmodernisme, ou de répondre à la question d'une éventuelle synthèse dialectique entre postmodernisme et modernisme. Il nous appartient encore moins de démontrer la véracité de l'hypothèse, établie par John Croft, de l'émergence d'une nouvelle mimesis esthétique. Néanmoins, une autre question se pose, s'élevant à la fois comme un moyen permettant de contourner l'aporie et comme un but réflexif en soi : quel rapport au passé observe-t-on aujourd'hui, depuis l'aube du XxI ${ }^{\mathrm{e}}$ siècle, dans un contexte actuel d'héritage moderniste ${ }^{11}$ et d'évolution esthétique post-postmoderniste ? Notre dessein

8 John Croft emprunte ces deux appellations à Jean-François Lyotard (Croft 2009, p. 29).

9 Croft se réfère à Giorgio Agamben qui effectue le constat d'une perte de contenu de l'art de la fin $\mathrm{du} \mathrm{xx}^{\mathrm{e}}$ siècle, due à la désorientation de l'homme contemporain face à l'Histoire, lointaine, indéchiffrable, démultipliée, fragmentaire.

10 «... [T] he castle of culture has now become e museum in which, on the one hand, the wealth of the past, in which man can in no way recognize himself, is accumulated to be offered to the aesthetic enjoyment of the members of the community, and on the other, this enjoyment is possible only through the alienation that deprives it of its immediate meaning and of its poietic capacity to open its space to man's action and knowledge »; notre traduction.

11 Nous reprenons à notre compte le titre de l'ouvrage de Heile et nous renvoyons à la citation suivante : "J'aimerais souligner [...] l'absence de continuité historique simple et de grand récit, reliant le modernisme "héroïque" du début du vingtième siècle aux avant-gardes de l'après-guerre ainsi qu'à leur successeurs supposés au début du vingt-et-unième siècle "; notre de traduction de cet extrait : "I wish to emphasize that [...] there is no simple historical continuity or grand narrative, linking the heroic, modernism of the early twentieth century with the post-war avant-gardes as well as their putative successors in the early twenty-first century» (Heile 2009, p. 4). 
ici est d'exposer et analyser des faits et des poïétiques afin de contribuer à répondre à la question d'une après-postmodernité en musique. Pourquoi, et comment, utiliser le matériau passé depuis les années 2000 ? Il nous semble qu'aujourd'hui encore, chaque œuvre devient une réponse particulière à la réponse primordiale du rapport à l'histoire et à la tradition.

Dans quelle mesure pouvons-nous vérifier la thèse défendue notamment par Björn Heile et ses co-auteurs selon laquelle le modernisme est un " enjeu constant, une entreprise sans fin » (Heile 2009, p. 3) ${ }^{12}$ ?

Nous reprendrons les différenciations binaires présentées dans le tableau précédent pour observer leurs applications potentielles dans le champ de la création contemporaine et leur rapport de force. Nous y ajouterons les catégories substantielles au modernisme définies par David Metzer et observables encore à l'orée du XXI siècle : d'une part, la posture investigatrice propre au compositeur moderniste, d'autre part, une poétique aspirant à réaliser des idéaux de pureté et d'ascèse impliquant notamment la prégnance $d u$ fragmentaire et du silence, et enfin une nouvelle recherche d'expression immédiate. David Metzer, bien qu'il les inclue dans une même démarche d'investigation, distingue la poétique de l'expressivité moderniste pour les motifs suivants :

La recherche dans les techniques compositionnelles a permis d'offrir des points de focalisation structurelle après le déclin du sérialisme, tandis que celle de l'expression a trouvé son accomplissement lorsqu'elle a répondu au désir de forger de puissants idiomes expressifs (Metzer 2009, p. 27) ${ }^{13}$.

Nous y associerons la catégorie essentielle, dans le cadre de cet article, propre à la nouvelle esthétique de la mimesis prônée, par John Croft, de la diffusion du geste d'écriture appartenant à un style passé dans la substance de l'œuvre contemporaine. Nous utiliserons enfin les catégories structuralistes et historicisées définies par Scarpetta et Nattiez explicitant le rapport au « code» langagier de l'histoire. Certaines sont propres à l'époque postmoderne, telles :

- la « régression » (Scarpetta 1985, p. 29), c'est-à-dire la remise au goût du jour de styles du passé (Nattiez 1993, p. 165), " précisément parce que périmé[s] » (Scarpetta 1985, p. 29).

- le «tri » subjectif (ibid., p. 30).

12 «Après tout, le fait même qu'il continue à nous préoccuper, montre que le modernisme est un enjeu constant, une affaire en suspens; quand est-ce que l'on en a fini avec le romantisme? "; notre traduction de cet extrait : "After all, the fact alone that it continues to exercise us shows that modernism is an ongoing concern, unfinished business; when did we last fight over romantiscism?» (Heile 2009, p. 3).

13 "The inquiry into states offered points of structural focus after the decline of serialism, whereas that into the act of expression has come into its own as it has satisfied the interest in forging forceful expressive idioms "; notre traduction. Metzer estime par ailleurs, et contrairement à Adorno, que l'expressivité peut exister sans qu'il y ait présence métaphorique et analogique d'un sujet dans le discours musical : "Finalement, l'expression peut exister par elle-même, et ne pas être nécessairement assignée à un sujet spécifique "; notre traduction de cet extrait : "Finally, the act of expression can stand by itself, not necessary assigned to a specific persona " (Metzer 2009, p. 24). 
Certaines sont constitutives du modernisme, telles :

- la « table rase » ou " avant-gardisme ", ou « novation » révolutionnaire chez Nattiez (1993, p. 165).

- ce que nous pourrions nommer 1'" extrapolation exclusive ", c'est-à-dire « l'appui sur un évènement majeur de [1']histoire, et n'en exploiter qu'une virtualité au détriment de toutes les autres »(Scarpetta 1985, p. 30).

- 1'assimilation innovante utilisant les profils objectivés et conceptualisés de la tradition : " concevoir [...] l'histoire du code non comme une accumulation figée d'œuvre mais comme un réservoir de virtualités - certaines actualisées, d'autres non; se situer non dans une logique du "coup", mais dans une temporalité de l'après-coup » (ibid.).

Certaines sont mixtes, pouvant être appliquées par les modernistes comme par les postmodernistes :

- 1'« académisme » (ibid.), ou « épigonisme » (Nattiez 1993, p. 165).

- 1'« accomplissement », ou " pousser à un haut degré de réussite les potentialités d'un genre » (ibid.).

Dans ce contexte, la problématique essentielle à laquelle cet article est dédié est non seulement la motivation pour laquelle, et la façon dont laquelle, un compositeur aujourd'hui introduit dans son œuvre des extraits de pages et des techniques compositionnelles héritées du passé, mais aussi la réponse apportée par les compositeurs actuels à la question de l'innovation. La problématique de la nouveauté demeure, une fois écartée ou non la conception moderniste de l'histoire comme un fardeau ou comme un modèle sans cesse à dépasser ou rejeter, en une révolte du fils contre le père. La dissolution de l'interdit d'interdire n'invalide pas l'interrogation sur les modes d'absorption et de réception de l'histoire. Comment être original si l'on choisit de se référer aux œuvres du passé, aux esthétiques et au matériau passés? Chez lesdits postmodernistes, des ponts sont établis entre les différentes époques et les langages. Berio composant sa Sinfonia en 1968 n'est pas un cas isolé. Bernd Aloïs Zimmermann dans son opéra Die Soldaten et dans la Musique pour les soupers du roi Ubu (1966) utilise la technique du collage d'extraits musicaux de la Renaissance. George Rochberg dans Nach Bach (1966) intègre des extraits retravaillés de la Partita $\mathrm{n}^{\circ} 6$ en mi mineur de Bach à une partition au langage atonal. Kagel écrit en 1969 sa Première Symphonie, qui emprunte à Beethoven, Chopin, Strauss, Grieg, Tchaïkovski, Haydn et au Dies Irae. Ces liaisons sont également des barrières : il s'agit de rendre un hommage aux maîtres tout en faisant valoir le fossé béant qui nous en sépare. Peter Maxwell Davis (né en 1934) intègre dans ses compositions la musique de la Renaissance, dont celle justement de Gesualdo à laquelle il fait subir des transformations techniques caractéristiques des années 1960 mettant l'emphase sur la distanciation historique entre les œuvres.

Face aux compositeurs créant, dans les années 2000, le nouveau à la source de l'ancien, il est pertinent de se demander si leurs attitudes continuent de refuser l'alternative entre la confrontation avec les « pères » et le reniement de ceux-ci par l'héritier, et si elles rejettent la démarche de négation des origines pratiquée par Darmstadt et les avant-gardistes. Sont-elles en quête de nouvelles narrativités et postures devant 
l'histoire, en gommant le temps unidimensionnel et unidirectionnel, et en faisant du présent un état multitemporel, conscientes que celui-ci est le carrefour d'histoires plurielles? Au contraire, assiste-t-on à l'affirmation d'un modernisme qui n'a plus besoin de se revendiquer comme tel, mais qui prend consciemment le contrepied du postmodernisme?

Nous apporterons des éléments de réponse à cette question par une étude ancrée au sein du niveau poiétique, fondant sa réflexion sur des entretiens avec deux compositeurs actuels, mais appartenant à des générations différentes : Michel Gonneville, né à Montréal en 1950, et Ofer Pelz, né en Israël en 1978, résidant à Montréal. Apparaissent ici deux paradigmes d'attitudes antithétiques à l'égard du passé : celui d'Ofer Pelz, pour lequel l'utilisation de matériaux passés ne peut être qu'une irrémédiable exception ou pratique conjoncturelle, et de Michel Gonneville, qui cultive une esthétique de la référence non déférente. Sur le plan méthodologique, afin de stimuler la réflexion, en guise de point de départ, un questionnaire leur a d'abord été remis, établi par nos soins en juin 2012, énonçant les questions suivantes :

- Quels sont pour vous le but de l'usage d'un matériau thématique, harmonique, rythmique, etc. ancien et déjà stylistiquement " parachevé ", ayant déjà acquis sa valeur esthétique, possédant déjà une signification interne dans une œuvre donnée et ayant donné lieu à une réception évoluant au fil de la réception historique?

- Est-ce un hommage de votre part?

- Souhaitez-vous créer un sens esthétique inédit pour cet extrait musical ancien, renouvelé en l'associant avec du nouveau?

- Pourquoi avez-vous choisi cet/ces extrait(s)-là en particulier? Est-ce parce qu'il(s) s'est (sont) un manifeste de votre canon de beauté?

- Désirez-vous en outre donner forme à, et montrer à l'auditeur votre propre " réception » du matériau passé que vous employez?

\section{Ofer Pelz, un nouveau moderniste}

Ofer Pelz, né en 1978, a reçu le Prix ACUM 2012, l'équivalent du Prix de la SOCAN en Israël, pour sa pièce Rewind, le Prix ACUM 2008 récompensant la meilleure création de 1'année pour sa pièce Equilibrium, le $2^{\mathrm{e}}$ Prix au Concours ACMF (Asian Contemporary Music Festival) en Corée du Sud, en 2010, et le Ernst Von Siemens Grant pour Do Bats eat Cats?. Sa musique est jouée régulièrement en Europe, aux États-Unis, au Canada ou en Israël, dans des festivals tels que La Biennale de Venise, MATA Festival (New York), Heidelberger Biennale für Neue Musik ou encore Nuova Consonanza (Rome).

Ofer Pelz défend une vision que l'on pourrait nommer moderniste, au sens érigé par Heile. Il admire en premier lieu Lutosławski, Ligeti, Grisey et Furrer. Il approuve et commente la distinction entre modernisme et postmodernisme établie avec esprit par John Rea, plus particulièrement ses assertions numérotées par ce dernier 12, 7 et 5 dans un ordre volontairement désordonné :

12) Le postmodernisme porte sur le souvenir tout en vous demandant avec coquetterie d'oublier. 
7) Parce qu'il pousse, le postmodernisme tend à rassembler une myriade d'artefacts éparpillés sur le site archéologique; il ne fait pas beaucoup de discrimination mais il a une conscience écologique. Le modernisme, parce qu'il tire, a une tendance à la séparation agressive, préférant les nouvelles constructions sur le site original et laissant aux environnementalistes le soin de se soucier des résultats une fois l'édifice terminé.

5) La postmodernité est une Femme, la modernité, un Homme. La postmodernité est monodie, mélodie, berceuse ; la modernité est contrepoint, des fois une jam session intensément criarde. Ainsi Mozart est une Femme, Bach, un Homme; Schubert, une Femme, Beethoven, un Homme; Clara Schumann, une Femme, Hildegaard von Bingen, un Homme (Rea 1997, p. 67).

La problématique de la référence au passé, dans laquelle John Rea distingue le choix radical d'oublier de celui du devoir de se souvenir, interpelle particulièrement Ofer Pelz. Celui-ci viserait idéalement le premier type de choix. Il envie ceux qui avaient la possibilité d'être ultra novateurs avant l'ère postmoderniste :

Quand j'ai commencé à m'intéresser à la musique "savante ", confie-t-il, j'étais autant attiré par la musique moderne $\mathrm{du} \mathrm{xx}^{\mathrm{e}}$ siècle que par la musique ancienne. Il y a beaucoup de liens entre les deux époques (on voit souvent des œuvres de ces périodes se côtoyer dans les programmes de concerts) (Ofer Pelz, entretien avec Liouba Bouscant, 10 octobre 2014).

Ofer Pelz est donc un compositeur qui croit en la métaphore, énoncée par Kandinsky dans Du spirituel dans l'art, du triangle avançant dans le temps, dont la pointe représente la créativité moderniste et avant-gardiste (Kandinsky [1911]1989, p. 61) ${ }^{14}$. Il est nécessaire, en effet, selon Pelz, d'avancer au sein du mouvement général de l'évolution des esthétiques et du langage dans l'histoire. Il faut s'abstenir de régresser et surtout, de faire du sur-place. Ofer Pelz se place, certes non pas à la pointe, mais vers l'angle supérieur du triangle de Kandinsky. La partie du prolongement écartelé des angles représente les styles et esthétiques situés avant les siens dans le temps, cependant dénués de classement ou de hiérarchie chronologique. La hiérarchie s'effectue sur le plan du goût. Le passé forme pour lui un tout global indifférencié auquel appartiennent indistinctement le plus proche comme le plus lointain des styles. Il dure indistinctement jusqu'à hier.

Il faut prendre acte que chez ce compositeur, le passé est un obstacle psychologique et une source d'anxiété morale. "On désire être unique, d'une part, et d'autre

14 «Un grand Triangle divisé en parties inégales, la plus petite et la plus aiguë dirigée vers le haut - un assez bon schéma de la vie spirituelle. Plus on descend, plus les sections du Triangle sont grandes, larges, spacieuses et hautes. Tout le Triangle avance et monte lentement, d'un mouvement à peine sensible et le point atteint "aujourd'hui" par le sommet du Triangle sera dépassé "demain" par la section suivante. Ceci veut dire que ce qui n'est aujourd'hui intelligible que pour la pointe extrême, et n'est pour le reste du Triangle qu'élucubrations incompréhensibles, sera demain, pour la seconde section, le contenu chargé d'émotion et de signification de sa vie spirituelle. » 
part, on peut nourrir des scrupules à se saisir et à s'approprier des inventions géniales qui ne proviennent pas de sa propre créativité ", déclare-t-il. Selon lui, "l'époque moderniste a en effet durablement légué pour héritage l'obligation d'être original, par l'innovation ». Cependant, Pelz ne " pense pas qu'il faille être absolument nouveau au XXI ${ }^{\mathrm{e}}$ siècle » et dénie le principe de la «table rase ». Il récupère le matériau d'autrui, malgré sa "mauvaise conscience morale ", arguant, contre l'éthique, non seulement que tout créateur est plus ou moins consciemment influencé par l'extérieur et le passé, mais, de surcroît, qu'il faut apprendre du passé et l'exploiter. Quand bien même cette intégration serait moralement assimilable au pillage, ce qui est exigible est que le compositeur ait conscience du vol, reconnaisse le propriétaire et bonifie son tribut. C'est à ce prix que s'opère la construction d'un style.

Cela n'implique absolument pas de tout imiter et de tout utiliser. Le compositeur doit demeurer critique et prendre position pour circonscrire son style.

Ofer Pelz, particulièrement réceptif à la réflexion 7) énoncée par John Rea renchérit sur la métaphore de l'architecture opposée à l'archéologie : le modernisme est en effet d'abord structuration du matériau et recherche de cohérence formelle. Ces caractéristiques impliquent un travail des parties et une transformation adéquate des éléments " étrangers " afin d'instaurer un rapport dialectique et dialogique entre les parties et le tout. C'est pourquoi Pelz se montre-t-il circonspect à l'égard de l'éclectisme et de l'usage des citations, qui par leur hétérogénéité, nuisent à l'unité formelle.

Par ailleurs, de l'association historicisée, valable pour le $\mathrm{xxI}^{\mathrm{e}}$ siècle mais non pour l'époque Renaissance et baroque, entre contrepoint et modernisme - voire avant-gardisme -, ainsi que de la relation d'identité posée entre effets de dissonance et modernisme effectuées par John Rea (1997, p. 67), jaillissent les idées d'une complexité et d'un défi lancé aux habitudes d'écoute. En effet, approuve Ofer Pelz, le modernisme implique à la fois une culture de la complexité et une musique élitiste, aspects rejetés par le postmodernisme.

L'œuvre d'Ofer Pelz, Moro lasso, composée en 2006 à partir du madrigal éponyme écrit en 1610 par Carlo Gesualdo, Moro Lasso al mio duolo à 5 voix, publié en 1611 dans le Sixième Livre de madrigaux, offre un très beau prototype de la démarche de ce compositeur. L'absence de hiérarchisation chronologique ou esthétique des œuvres du passé frappe. Ofer Pelz s'approprie et traite ce matériau préformé de la Renaissance sans tenir compte de son contexte et de ses implications harmoniques et stylistiques, mais en retenant uniquement les "gestes " d'écriture, dans le sens utilisé par John Croft : le rythme, la dissonance, les tournures mélodiques, la texture de contrepoint. Sa démarche consiste à relier ses propres procédés d'écriture à ceux d'œuvres passées. Il explique le choix de cette pièce en ces termes :

Quand j'ai écouté le madrigal de Gesualdo Moro Lasso al mio duolo pour la première fois, immédiatement j'ai eu l'idée d'établir une conversation avec cette pièce. En écoutant la pièce originale, j'ai été très surpris par son originalité - surtout par les accords du début - qui peut même être perçue comme de la musique d'aujourd'hui, et qui reflète une modernité, voire une avant-garde, qui sont représentatives de la Renaissance. Un autre élément qui m'a beaucoup attiré vers la partition de Gesualdo est la personnalité et la 
biographie du compositeur, aspects qui occupent une place importante dans la perception de la pièce par un regard historique (Ofer Pelz répondant au questionnaire de Liouba Bouscant, 2012).

Ofer Pelz s'emploie ainsi à mettre en exergue le modernisme d'un matériau ancien pour le connecter à sa propre modernité. À cette fin, il replace un matériau qui appartenait à un univers de repères esthétiques, culturels spécifique, dans un autre système de signification. Il impose ensuite une évolution forcée et expresse au madrigal de Gesualdo, depuis la Renaissance au XXI siècle ; il lui fait faire, en quelque sorte, un bond dans le temps. Par conséquent, il ne s'agit ni d'une simple citation, ni d'une forme thème et variations, ni d'une simple transmutation contemporaine d'une expression ancienne, mais d'une réactualisation de l'attribut moderniste d'une œuvre passée. Ofer Pelz, qui possède un champ historique et des outils et références contemporains, veut, selon ses termes, procéder à une « application de la pensée contemporaine sur une pensée ancienne » (ibid.).

On peut donc s'arrêter à considérer qu'il s'agit là d'une récupération de l'histoire. Cependant, l'objet du passé n'en sort pas indemne : c'est un autre qui est formé. Toutefois, l'illusion au début d'un collage ou d'une citation et d'une rémanence du passé est créée par le compositeur : "la pièce commence exactement comme le madrigal de Gesualdo, et l'auditeur ne sait pas s'il s'agit de la musique originale ou s'il s'agit d'une création ». Puis, au chiffre $B$, la partie nouvellement créée par Pelz commence.

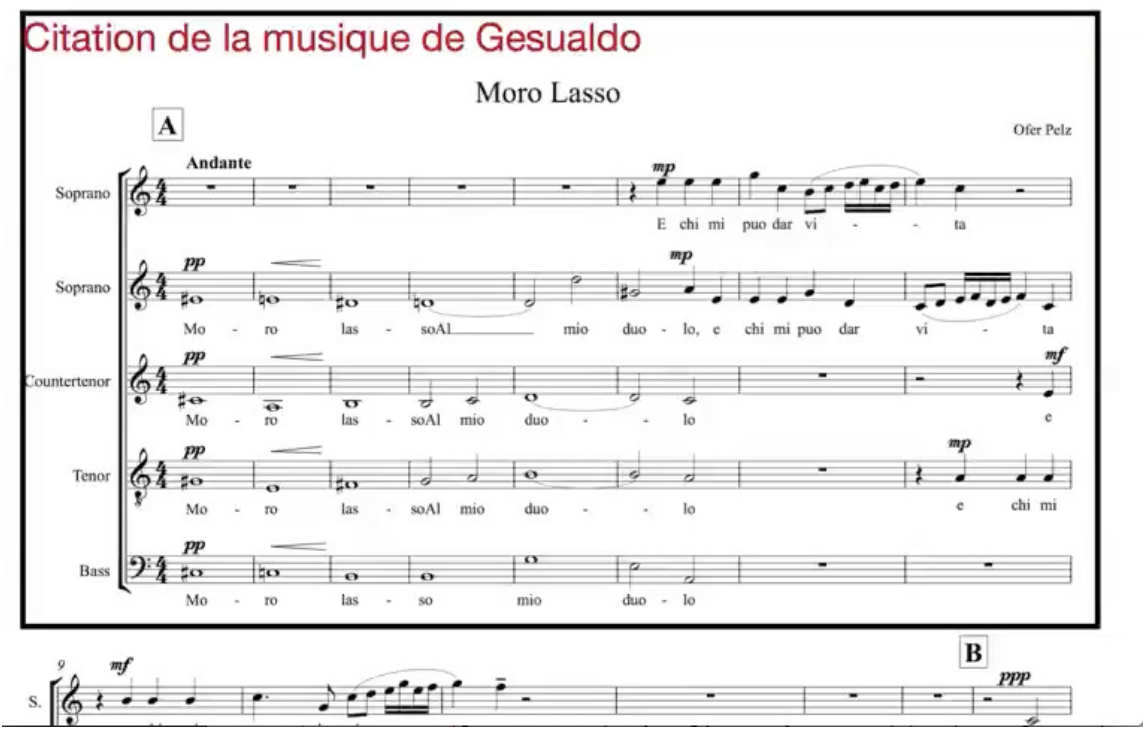

Exemple musical 1 : Moro Lasso, Ofer Pelz, mesures 1 à 24.

Une coupure sémiotique est donc effectuée dans un premier temps. La musique de Gesualdo est extraite de son contexte et introduite dans le XxI ${ }^{\mathrm{e}}$ siècle. La démarche de Marcel Duchamp, justement " parangon de l'avant-garde " comme le fait remarquer Scarpetta (1985, p. 18) est volontiers invoquée par Ofer Pelz. Celui-ci aussi a voulu 
recréer une nouvelle dimension esthétique en utilisant un matériau ready-made et en jouant avec les intersections des horizons d'attente au niveau de la réception.

Mais cette extraction historique est en réalité recréation d'un nouvel objet extirpé de son contexte, démarche avant tout moderniste voire d'avant-garde. En effet, Ofer Pelz procède à un travail en profondeur à partir des caractéristiques du matériau historique. On trouve ici l'application de la métaphore moderniste de l'architecture forgée par John Rea, en opposition à la préservation d' " artefacts » intacts propre à l'archéologie assimilée au postmodernisme.

Moro Lasso comporte plusieurs sections qui poursuivent des relations distinctes avec le madrigal original. Le graphique ci-après est une représentation structurelle des niveaux de similitude avec le madrigal de Gesualdo.

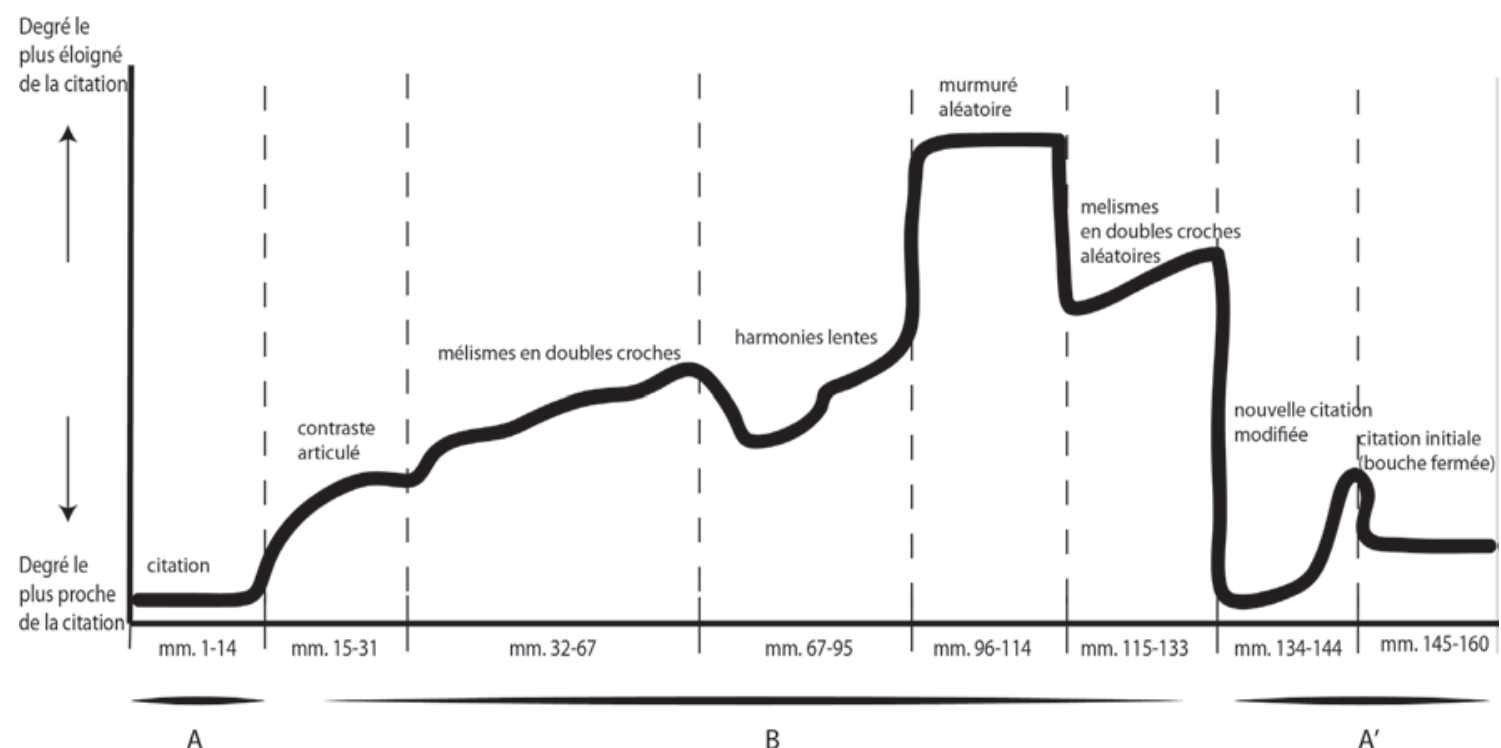

Figure 1 : Variations des degrés de conformité avec le matériau historique (Gesualdo) dans Moro Lasso d'Ofer Pelz.

Nous pouvons remarquer, à la lecture de cette courbe, qu'au début l'œuvre de Pelz est très proche du madrigal de Gesualdo, puis que vers le milieu, elle s'en éloigne. Elle s'en rapproche de nouveau vers la fin. Nous sommes par conséquent en présence d'un processus formel ternaire $A-B-A$ ', où le $A$ cultive une proximité avec le madrigal d'origine et utilise des citations, et le $B$ consiste en une exploitation plus libre des éléments tirés du madrigal.

Si le madrigal de Gesualdo est traité avec un style et un langage contemporains, les caractéristiques d'écriture et du genre du madrigal d'origine demeurent.

Les mélismes en doubles croches chez Gesualdo entendus vers la fin de la citation, sont en effet exploités largement dans la pièce, par la répétition et le développement, comme une étude portant sur le motif mélismatique. Ceci crée une sorte de continuum en doubles croches avec des moments d'accentuation. 


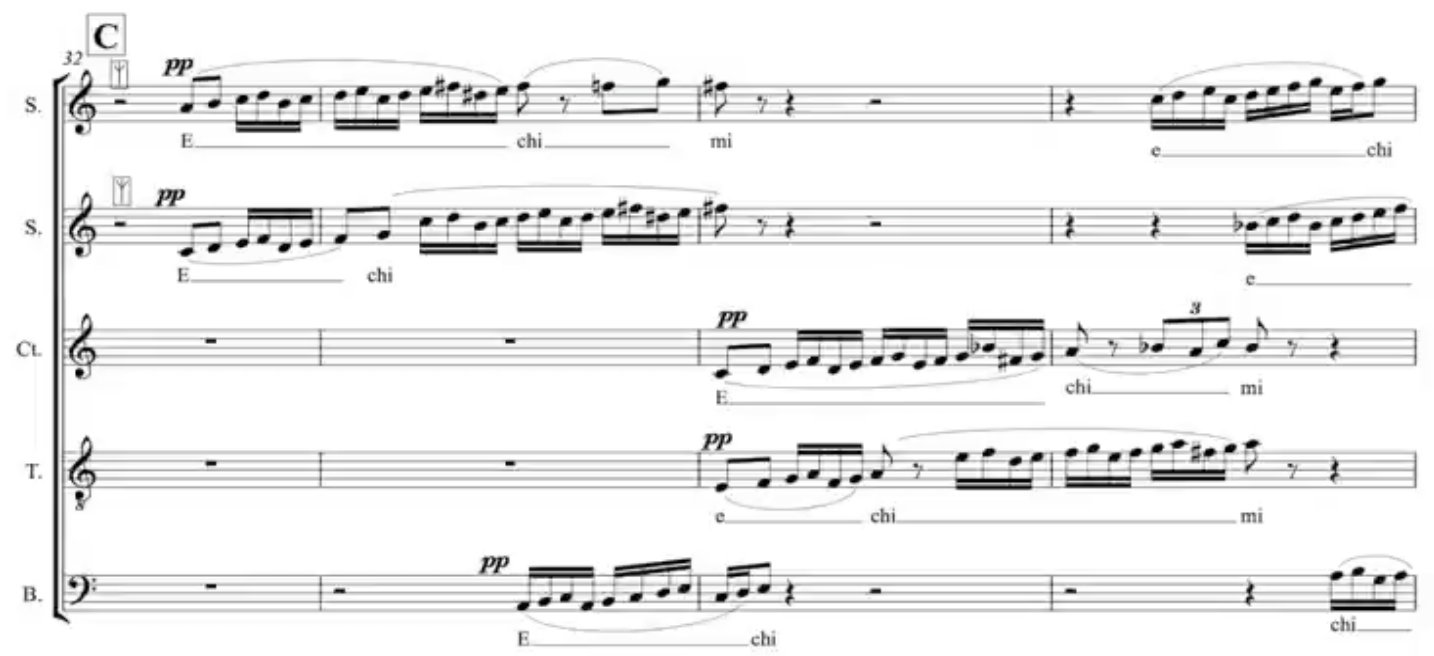

Exemple musical 2 : Moro Lasso, Ofer Pelz, mesures 32 à 38.

Les mélismes réapparaissent plus loin, dans un langage plus dissonant et atonal. Ils s'emballent en un rythme aléatoire. On parvient à ce moment-là au climax de l'œuvre.

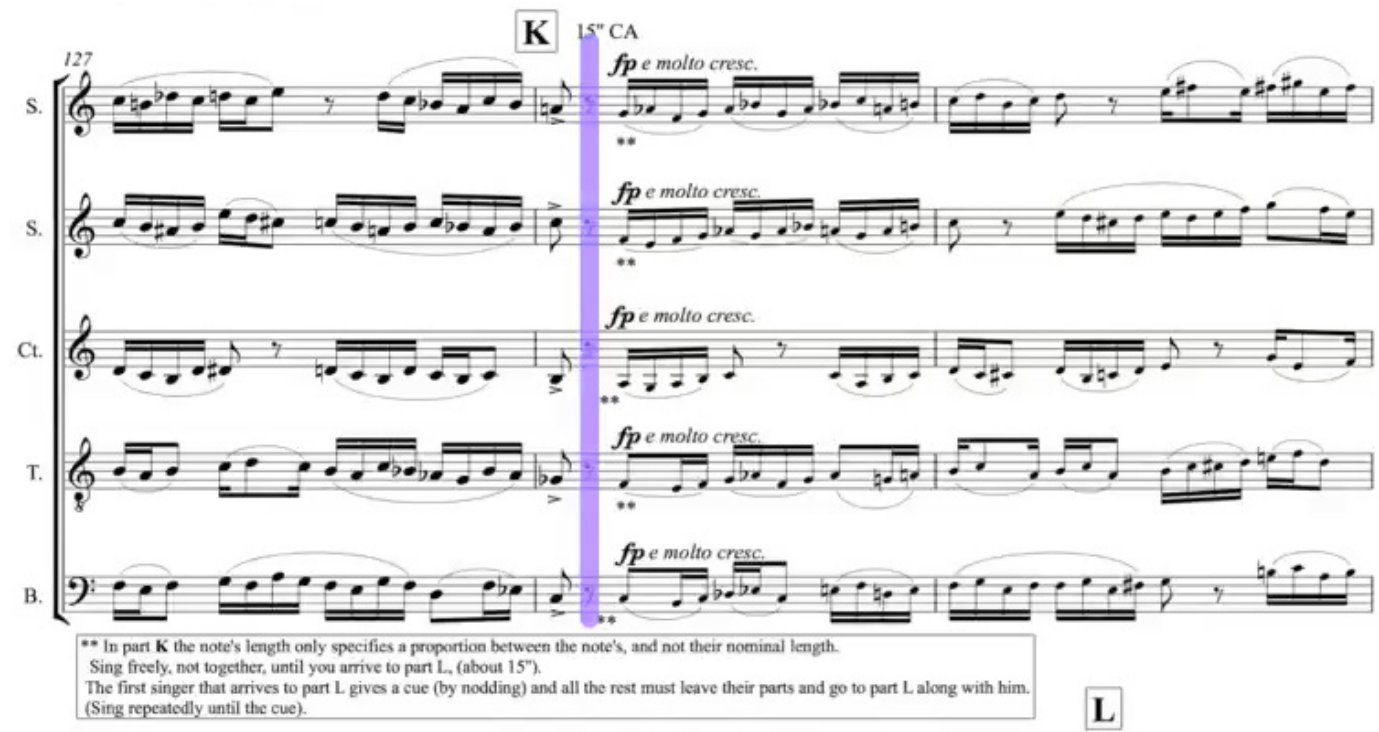

Exemple musical 3 : Moro Lasso, Ofer Pelz, mesures 128 à 132.

Dans la section qui suit, conservant uniquement les paroles de Gesualdo, les chanteurs murmurent et utilisent le Sprechgesang. Nous pouvons entendre dans l'extrait ci-après la fin du passage lent et le début du passage «parlé ». 


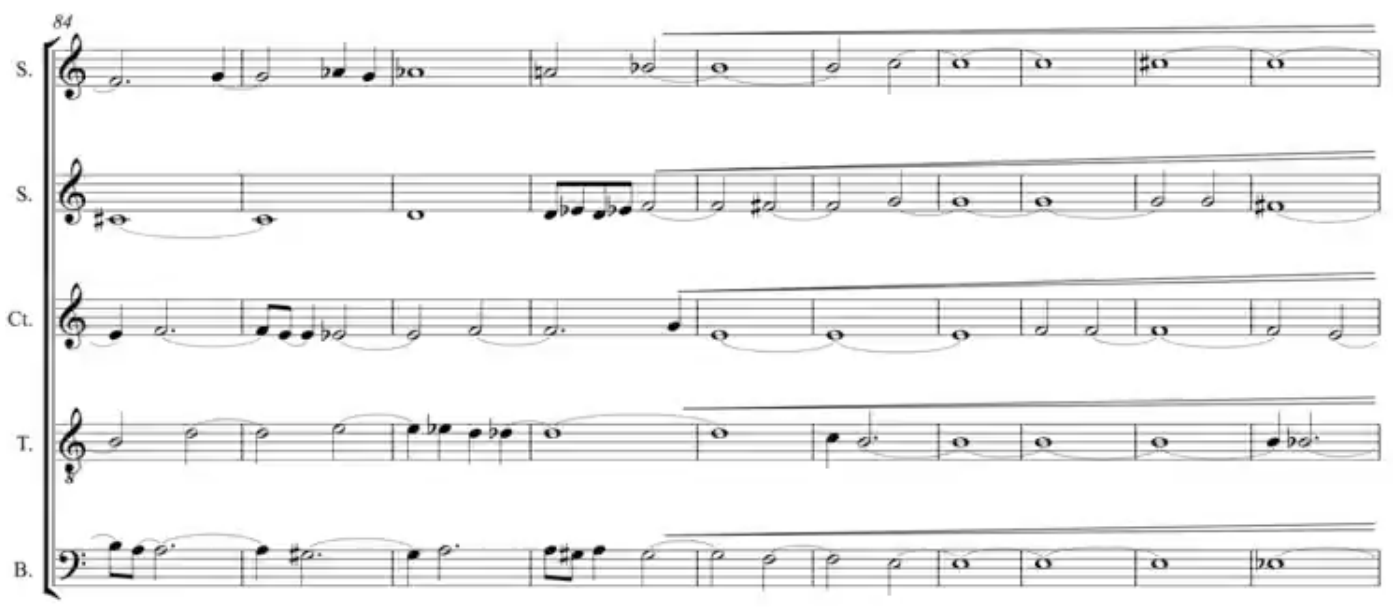

H $30^{\circ} \mathrm{CA}$

Exemple musical 4 : Moro Lasso, Ofer Pelz, mesures 87 à 114.

Pour Ofer Pelz, il importe de traiter des caractéristiques communes avec Gesualdo, en l'occurrence le mélisme, comme nous venons de le faire entendre, mais aussi la dissonance, selon sa propre esthétique et son propre langage. Ainsi le compositeur établit un lien entre la saturation des dissonances dans l'œuvre de Gesualdo et l'émancipation de la dissonance dont il se considère un héritier au XXI ${ }^{e}$ siècle. En effet, outre son insistance sur les similitudes des aspects horizontaux mélodico-rythmiques, il attire l'attention sur les points communs entre les dimensions verticales des deux pièces vocales : la section centrale, qui exerce un parallèle avec le tempo lent du madrigal d'origine, ainsi que les accords, où un travail harmonique est fait, notamment l'introduction de clusters. Cependant, des expressions contemporaines sont utilisées, tel un vibrato exagéré, qui n'existe pas chez Gesualdo.

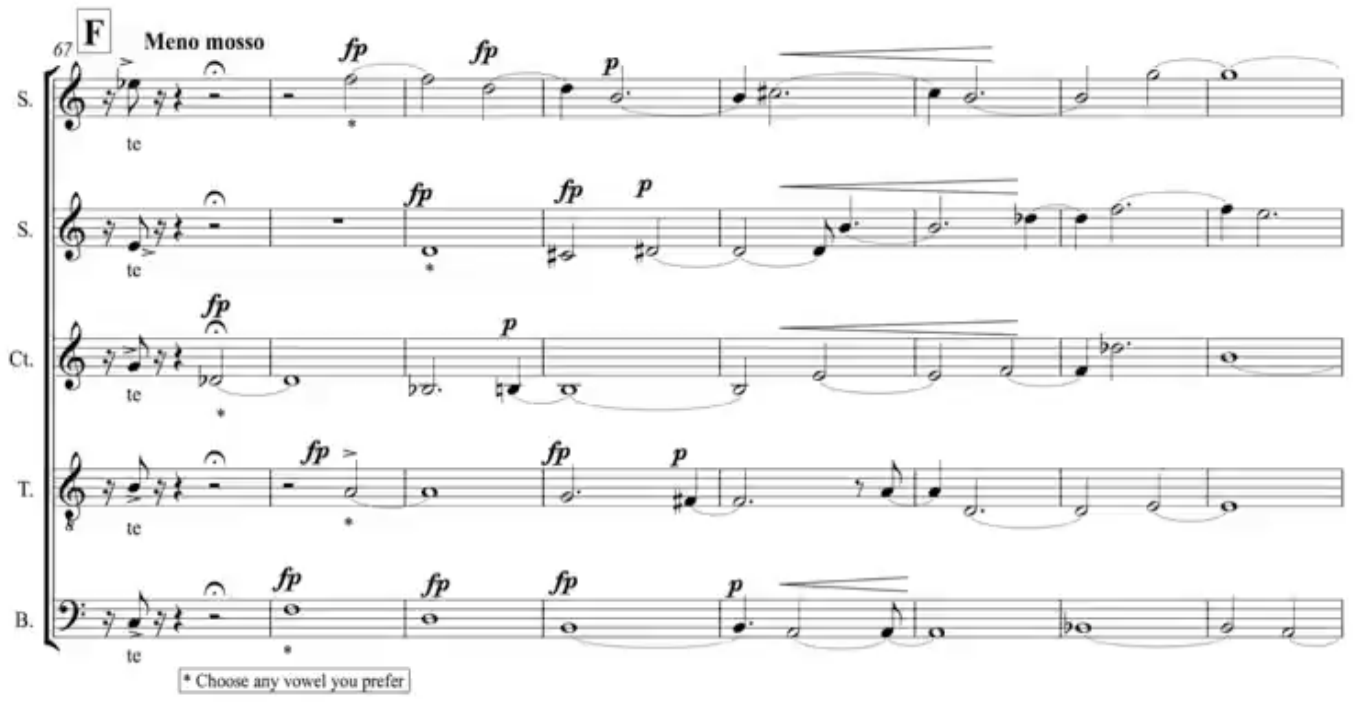

Exemple musical 5 : Moro Lasso, Ofer Pelz, mesures 67 à 86. 
Le madrigal se termine par la citation polyphonique de Gesualdo initiale, chantée bouche fermée. La citation se répète en boucle, mais à chaque fois une nouvelle voix change légèrement de conduite mélodique, créant une sorte de mutation de l'expression originale.

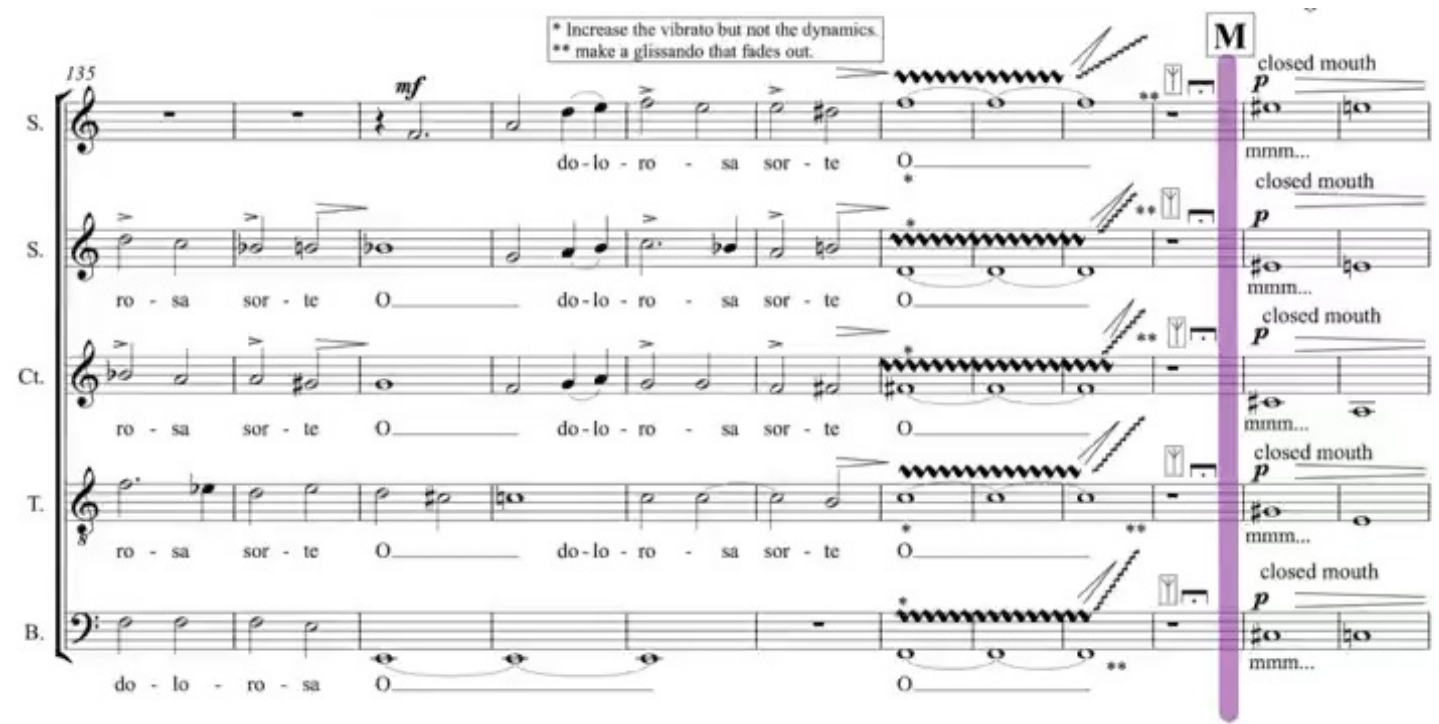

Exemple musical 6: Moro Lasso, Ofer Pelz, mesures 145 à 160 (fin).

Cette mise en confrontation par Ofer Pelz d'historicités éloignées, soucieuse de créer des ponts et de les injecter en profondeur dans l'écriture, tient d'une attitude moderniste, puisqu'elle ne pratique pas une technique de collage, se fonde sur l'évolution et le positivisme, et revendique un modernisme structurel rémanent dans l'histoire. En effet, Pelz appose sa pensée sur celle de Gesualdo en particulier, ce qui produit une synthèse des deux modernismes à partir de filtres contemporains. 


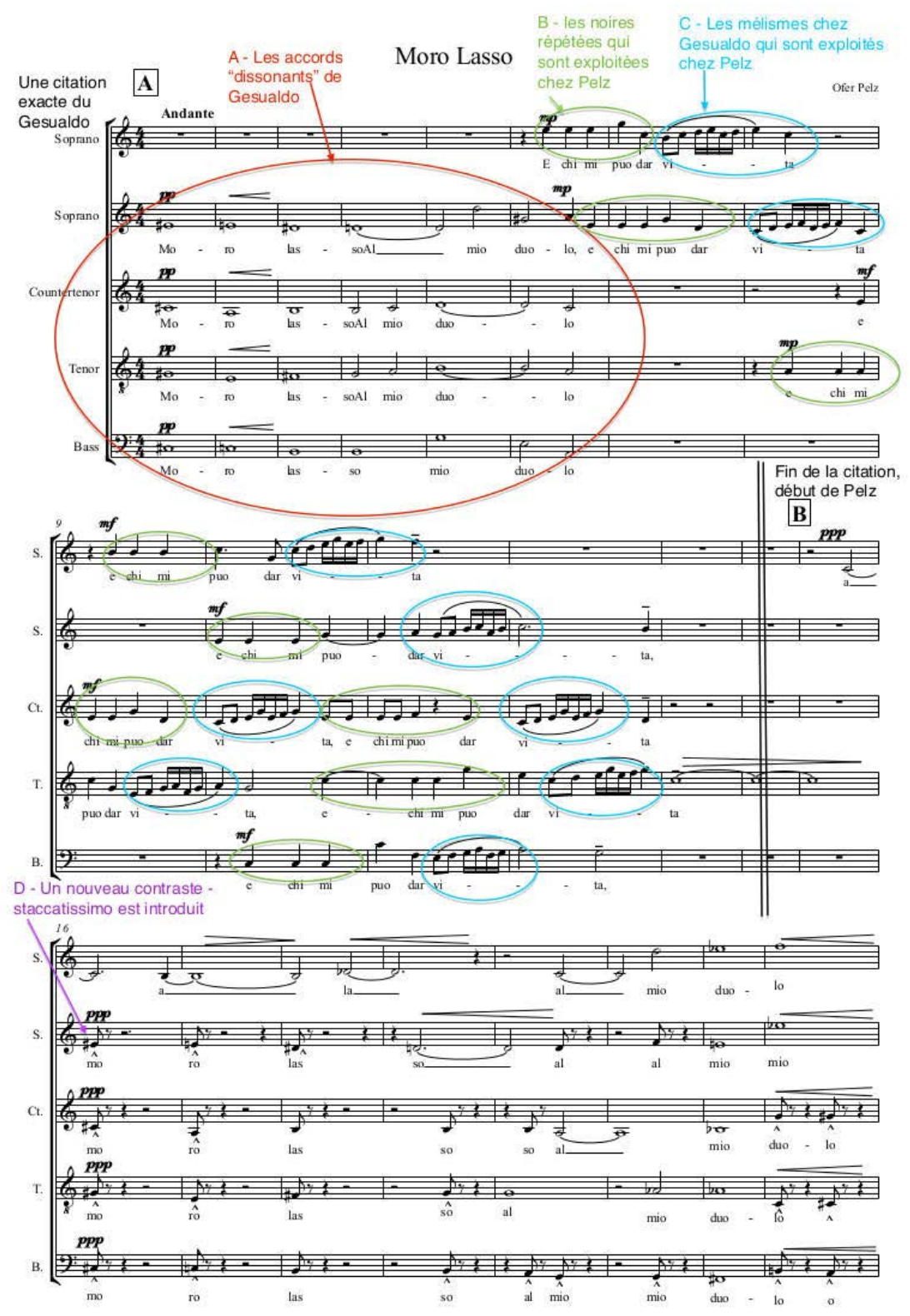

Exemple musical 7 : Moro Lasso, Ofer Pelz, pièce intégrale (voir en annexe la partition annotée de cette pièce).

Michel GonNEVILLE : LE « CORPS À CORPS » AVEC L'HÉRITAGE PASSÉ

Michel Gonneville a obtenu le Prix Serge-Garant 1994 de la Fondation ÉmileNelligan pourl'ensemble de son œuvre. Sa pièce Chute/Parachute, œuvre recommandée à la Tribune internationale des compositeurs, a été diffusée dans plus de 27 pays. Il a composé pour plusieurs ensembles et solistes québécois et étrangers. Ses œuvres ont été diffusées en concert et à la radio en France, en Belgique, en Angleterre, en Allemagne, au Mexique, etc.

Michel Gonneville mène, selon ses termes, un « corps à corps » avec l'héritage passé. Il se plaît à dialoguer avec l'histoire plutôt que de la rejeter. Il se positionne volontai- 
rement par rapport aux prédécesseurs et parle en termes d'évolution, de succession, de confrontation ouverte avec les maîtres. Son défi est de choisir sa propre voie. Il revendique une déférence mêlée d'une « certaine irrévérence par rapport [aux œuvres du passé] ». Il " ne pourra sentir qu'il fait partie de ce mystérieux mouvement de la création musicale qu'à cette condition de se retrouver d'égal à égal avec les maîtres du passé (sans égards aux moyens techniques, qui sont forcément autres), de sentir qu'il participe de la même joie créatrice, du même effort créateur qu'eux, mais à son époque propre » (Michel Gonneville répondant au questionnaire de Liouba Bouscant, 2012).

Michel Gonneville a toujours désiré passer outre les interdits et injonctions modernistes, tels que l'usage de la non-périodicité, l'athématisme, l'absence de référence à la tradition. Cependant sa réticence à être étiqueté " postmoderniste » provient de sa conception compositionnelle tributaire d'une recherche, d'une quête de l'inouii. Néanmoins, si Gonneville se réfère à Rimbaud dont il modifie le mot d'ordre " il faut absolument être moderne » en " il faut être absolument nouveau », ce nouveau, selon lui, peut naître du familier et de l'héritage des pères.

Force nous est de constater que la poursuite d'une démarche narrative interne et propre à l'œuvre, que Gonneville définit comme " raconter autre chose » à partir de la tradition, participe de la micronarrativité symptomatique de la période postmoderniste qui a fait éclater la notion de grands récits, selon la théorisation de Lyotard. En cela, la démarche créatrice de Gonneville traduit une mixité d'attitudes où domine le modernisme : tout à la fois l'expression d'un anti-avant-gardisme et la volonté de conservation de traditions, la pratique d'une micronarrativité de type postmoderniste, mais une dominante moderniste, par une aspiration à innover sans cesse et dépasser les prédécesseurs sur le plan de la cohérence formelle.

Contrairement à Ofer Pelz qui s'est essayé une unique fois à la citation et à l'emprunt, Michel Gonneville systématise cette démarche et l'ancre dans sa poétique. Il utilise ces procédés dans de nombreuses œuvres, selon plusieurs niveaux de reconnaissance du matériau cité. Les matériaux empruntés sont majoritairement issus du patrimoine savant, et plus particulièrement, d'œuvres de Haydn, Brahms, Chopin, Messiaen, Stravinsky, Bach, Mozart, Serge Garant, Gilles Tremblay. Voici les principales œuvres composées à partir de ce procédé compositionnel, de la plus récente à la plus ancienne :

1- Couple moteur, 2012 (Haydn et Brahms)

2- Couple au repos, 2011 (Chopin)

3- Volées. Carillons d'oiseaux, 2010 (Messiaen et Gilles Tremblay)

4- Trois scènes de couple, 2009 (Stravinsky)

5- Relais Papillons, 2009 (Chopin)

6- Browsing Agon, 2008 (Stravinsky)

7- Quatuor Rosemont. Inventions locales, 2007 (Chopin, Gilles Vigneault, heavy metal)

8- ... L'oiseau du cri à la quête des ailes..., 2004 (Messiaen)

9- Suivre la trace, perdre le fil, 2000 (hymne grégorienne)

10- Hinauf, dem Bach entlang, 1996 (Bach)

11- Montée de printemps, 1996 (Stravinsky)

12- Attiré vers le haut par le menu, 1995 (Mozart) 


\section{3- Approches, 1991 (Serge Garant) \\ 14- Se abrasa lumbre con lumbre, 1986 (Stravinsky) \\ Et en projet : Le petit théâtre de monsieur Pichon (Chopin)}

Michel Gonneville explique les raisons de ses choix d'extraits :

$[\mathrm{M}]$ es motivations [...] sont très variées : ils proviennent de pièces qui me poursuivent depuis plusieurs années, parfois depuis ma jeunesse; ou encore de pièces emblématiques pour un instrument. Il s'agit parfois de concordance expressive singulière du matériau emprunté avec ce qu'évoque le matériau plus personnel; ou de parenté matérielle étroite, avec le même type d'harmonie, par exemple (Michel Gonneville répondant au questionnaire de Liouba Bouscant, 2012).

Michel Gonneville estime avoir tiré de Cage la leçon selon laquelle il faut savoir déceler la beauté partout. Ce canon, tout en étant le résultat d'une saisie du Beau dans la diversité la plus grande, toute époque et tout style inclus, obéit plutôt au principe de « tri » sélectif arbitraire (Scarpetta 1985, p. 29). Gonneville continue en effet, somme toute, à faire valoir un canon personnel. Son panthéon réunit au sommet Stravinsky, Chopin, Bach et Ravel.

Comme Ofer Pelz, Michel Gonneville transforme le matériau emprunté au passé, selon une démarche " moderniste " constructiviste architecturale, selon la métaphore employée par John Rea (Rea 1997, p. 67). Son but, lors de l'élaboration thématique, n'est pas de citer, mais de retravailler. Il emprunte et traite sous forme de paraphrases, d'allusions, de métamorphoses. Il faut que ce matériau soit peu reconnaissable pour qu'il y ait un contre-pied. C'est pourquoi Michel Gonneville n'emploie pas les citations exactes de façon structurelle : celles-ci demeurent plutôt rares et sont souvent fugitives. Il désire constituer un objet musical personnel ayant un sens complet en lui-même. Selon lui, le sens esthétique d'une opération de citation ne peut se séparer du sens nouveau de l'œuvre qui l'intègre:

La citation plus ou moins reconnaissable peut ajouter une dimension culturelle, historique supplémentaire à la lecture de l'œuvre. Parfois, la pièce citée a servi carrément de matériau de départ sur lequel ont été opérées une foule de transformations. Mais le véritable défi est plutôt d'intégrer cette citation de façon à ce qu'elle serve le propos général de l'œuvre qui la cite. Le sens de l'œuvre intégratrice s'amplifie des références que traînent avec elles les citations, mais en même temps, elle peut détourner ces références, voire même forcer l'auditeur à les négliger pour ne retenir du matériau cité que ses rapports matériels (mélodique, harmonique, rythmique, textural, formel, etc.) avec le matériau original qui l'entoure (Michel Gonneville répondant au questionnaire de Liouba Bouscant, 2012).

Le matériau passé est ainsi le point de départ qui inspire, qui fait l'objet d'une réappropriation. Michel Gonneville impose au matériau passé une intégration dans son propre univers. Il procède par des sortes de variations amplifiées et d'extrapolations à partir d'un thème passé, alimentant l'œuvre. 
L'oiseau du cri à la quête des ailes... (2004)

L'oiseau du cri à la quête des ailes... (2004) offre un exemple concret d'œuvre « intégratrice ». Un thème de Messiaen inaugure l'œuvre, cité textuellement. Ensuite, la phrase est répétée, déformée. Cette déformation offre la thématique de départ.
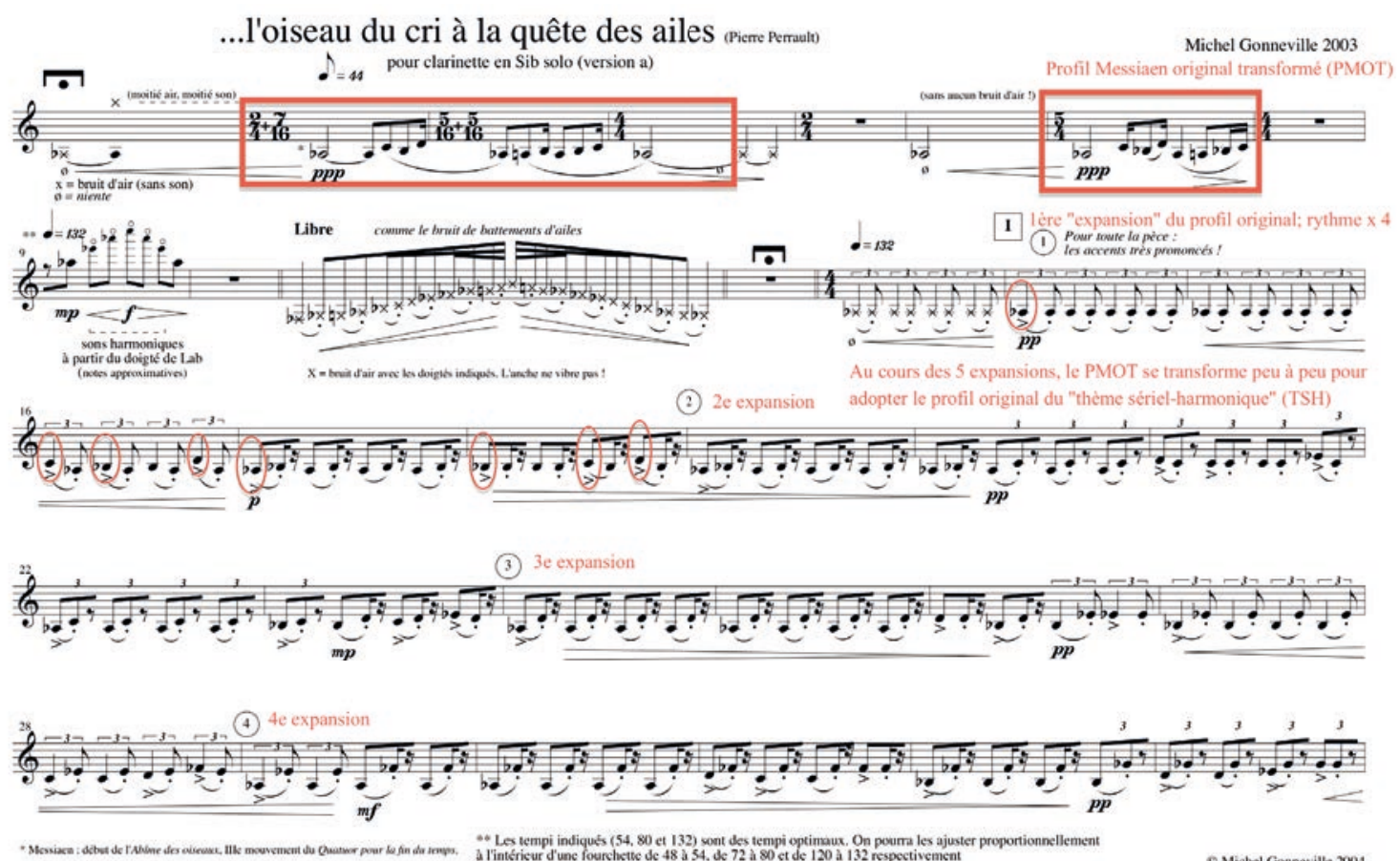

Exemple musical 8: L'oiseau du cri à la quête des ailes..., Michel Gonneville, mesures 1 à 33.

À partir du chiffre I, les accents font entendre la mélodie déformée.
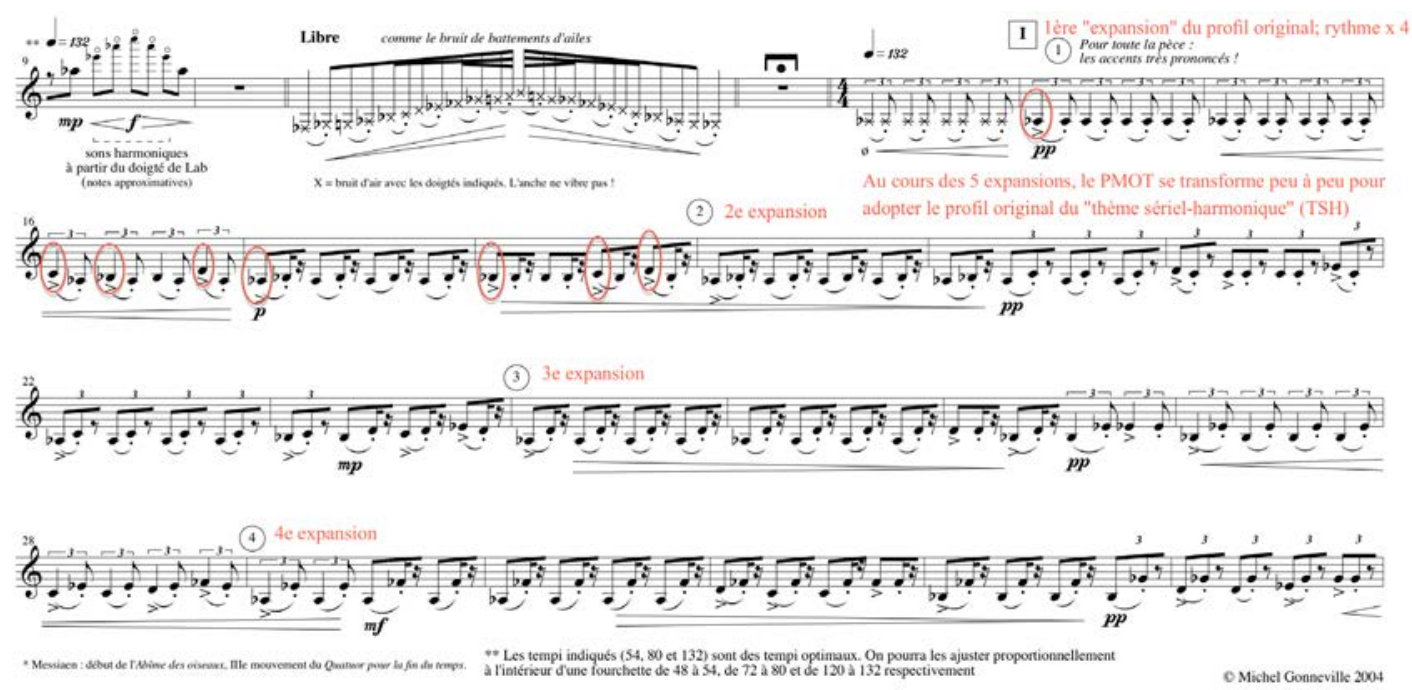

Exemple musical 9 : L'oiseau du cri à la quête des ailes..., Michel Gonneville, mesures 9 à 33. 
$\mathrm{Au}$ chiffre II, apparaît un contrepoint virtuel où les accents donnent le profil mélodique en augmentation intervallique à 2 voix.

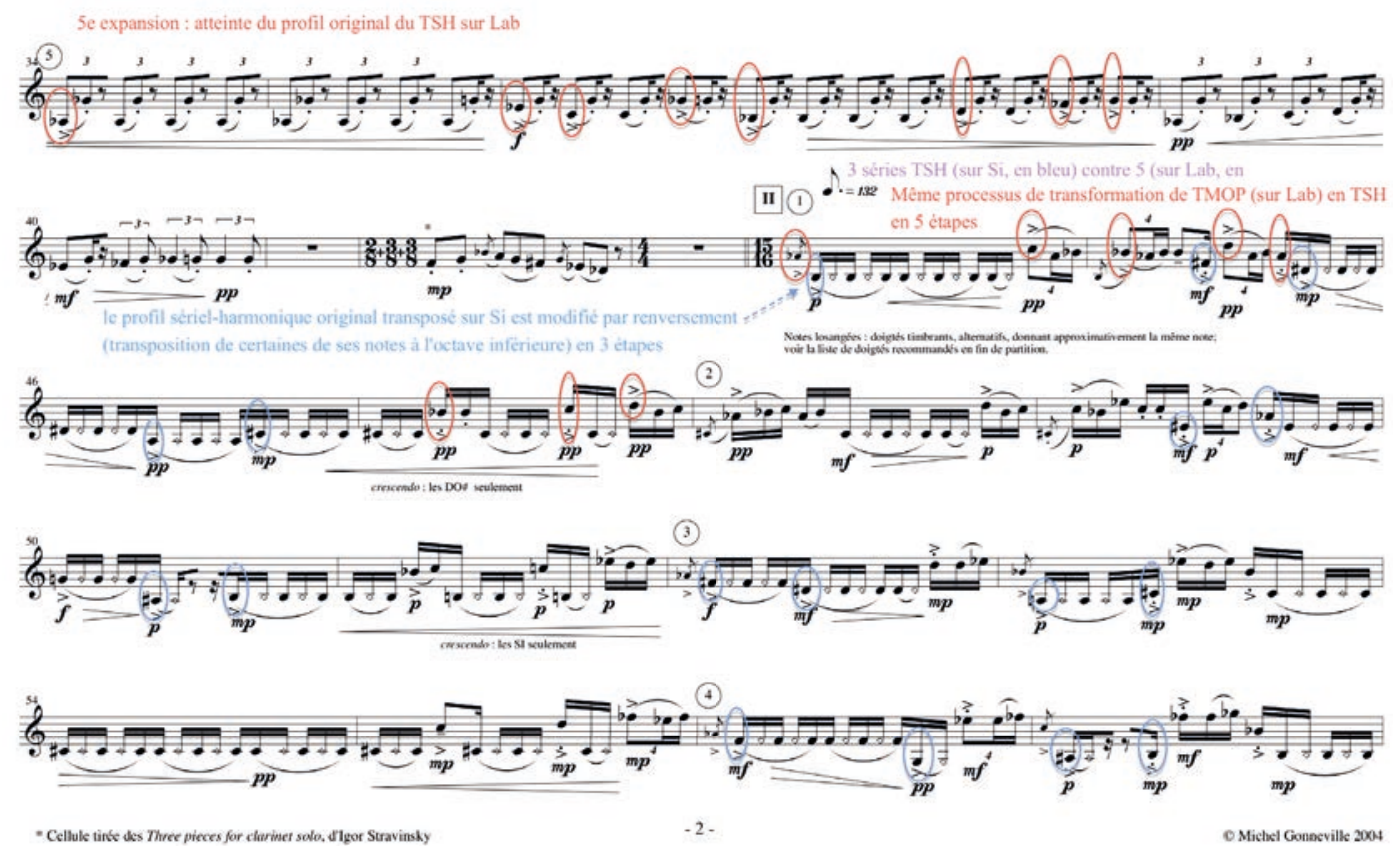

Exemple musical 10: L'oiseau du cri à la quête des ailes..., Michel Gonneville, mesures 34 à 57.

Petit à petit, le profil mélodique du thème de Messiaen, par anamorphose, adopte celui, caractéristique, d'un autre thème intrinsèquement lié au mode de prédilection de Michel Gonneville, le mode acoustique : do-ré-mi-fa dièse-sol-la bémol-si bémolsi bécarre.

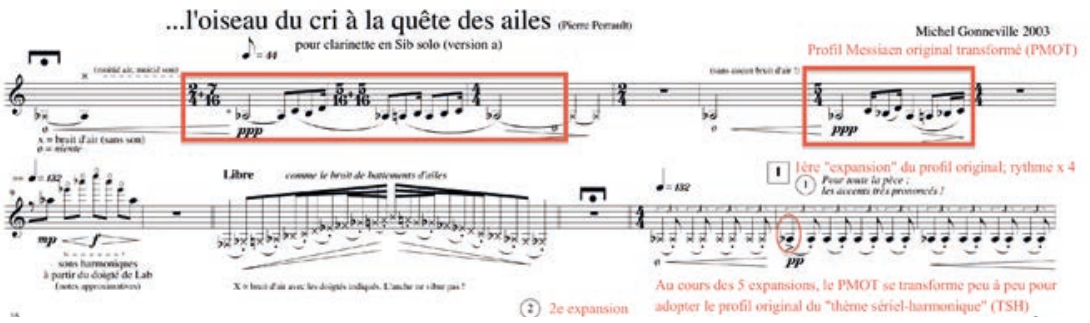

Exemple musical 11 : L'oiseau du cri à la quête des ailes..., Michel Gonneville, pièce intégrale.

En empruntant un matériau à l'héritage savant, Gonneville n'opère pas de remise en question esthétique critique. Mais il se place dans la continuité historique en affirmant sa propre identité.

Couple au repos (2011)

Un autre aspect important de l'emprunt à considérer réside dans le degré d'identité avec la source. Chez Gonneville, lorsque ce degré est faible, l'emprunt s'explique comme un point de départ compositionnel associé à la volonté de s'inscrire en filigrane dans la tradition et permet encore de se placer dans le sillage des maîtres. 
Ainsi, dans Couple au repos, par exemple, le compositeur prend pour point de départ de référence et compositionnel le largo de la Sonate pour violoncelle et piano opus 65 de Chopin.

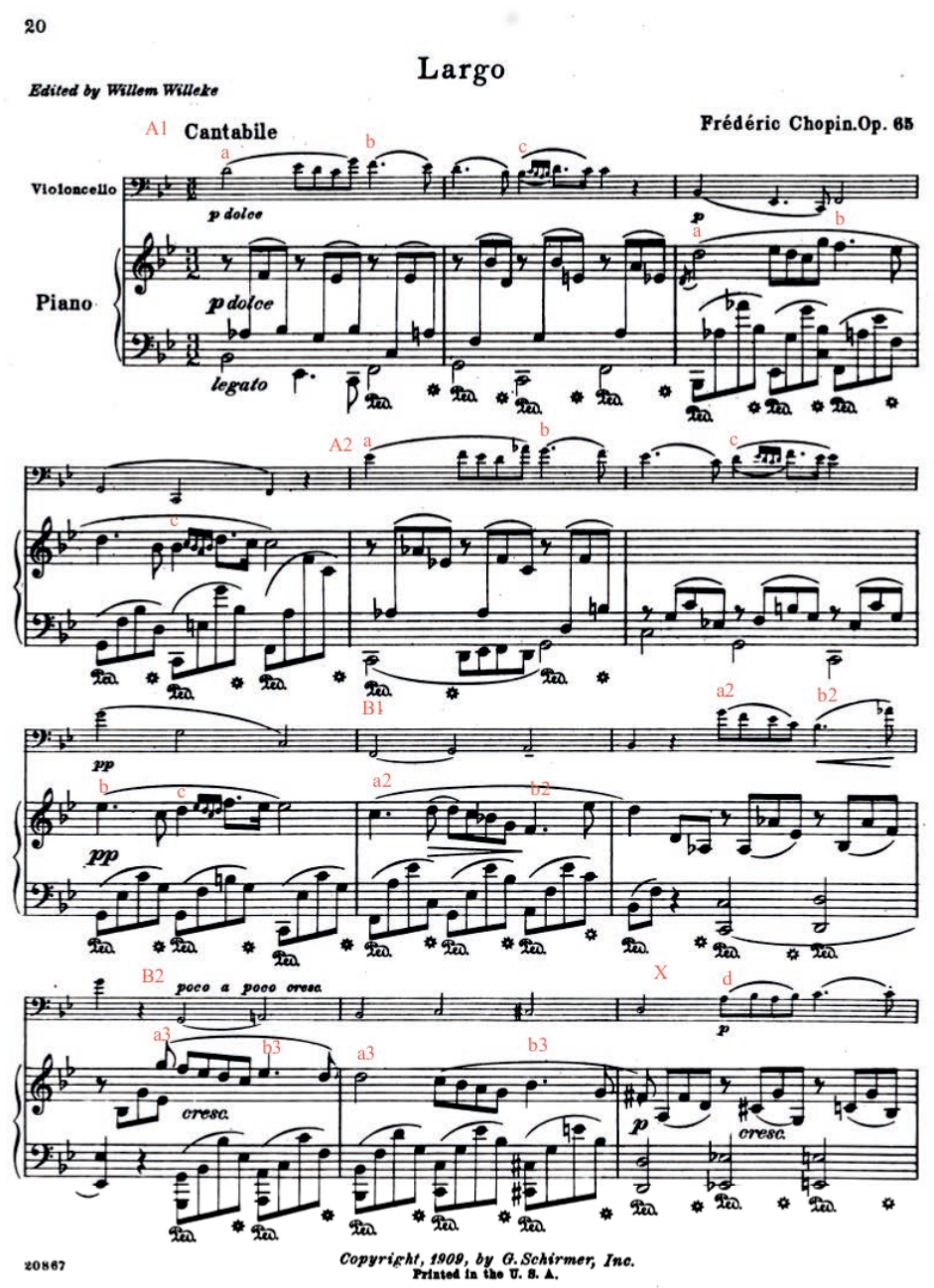

Exemple musical 12 : Sonate pour violoncelle et piano en sol mineur, op. 65, Frédéric Chopin, Largo, mesures 1 à 12 .

Exemple musical 13 : Sonate pour violoncelle et piano en sol mineur, op. 65, Frédéric Chopin (Maria Joao Pires et Pavel Gomziakov, 22 minutes 45 secondes).

Michel Gonneville maintient le rythme harmonique propre à l'œuvre-source. Cependant, opérant une disjonction de la mélodie et du rythme du thème écrit par Chopin, il en sectionne et distribue par rotation les hauteurs et conserve la structure rythmique, pour créer un nouveau thème. Ainsi, dans l'exemple qui suit, le rythme de la première phrase de Chopin (mesures 1 et 2) est, chez M. Gonneville, joué en alternance par le violoncelle et le piano (notes encadrées, mesures 3 à 9 - cf. la lettre $r$ minuscule), alors que le profil mélodique de cette même phrase de Chopin donne lieu, chez Gonneville, à une rotation à partir de la mesure 8 (au piano, cf. la lettre $m$ minuscule ; profil complet : mesures 8 et 9 puis mesures 3 à 7). 


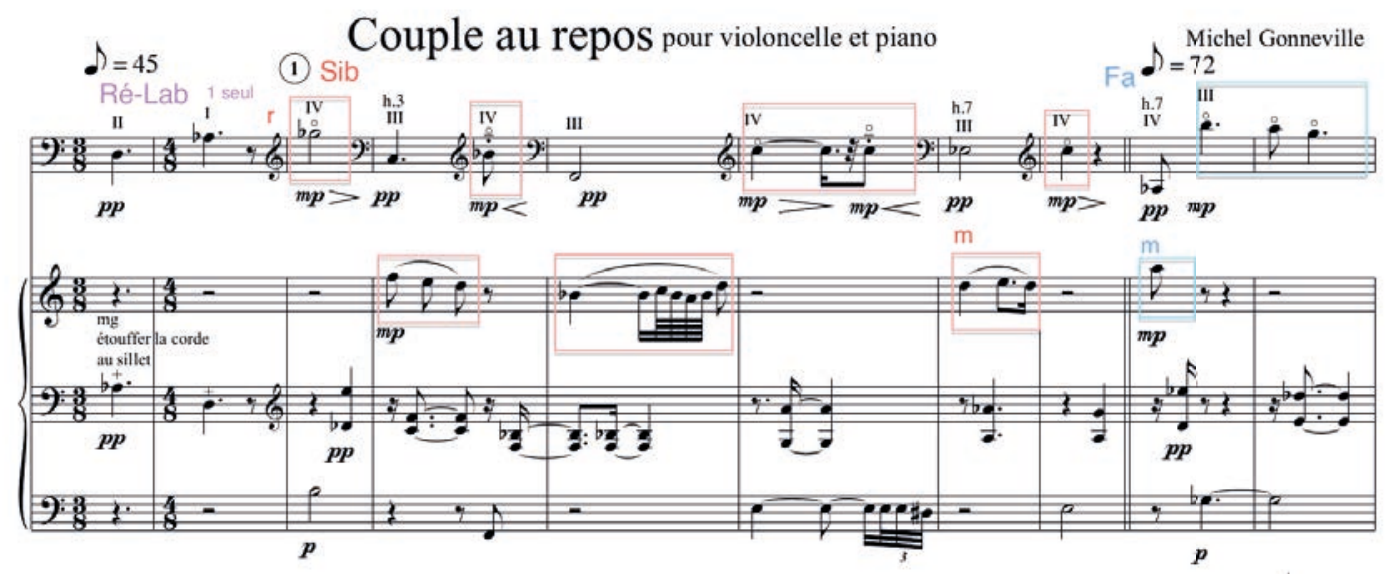

Exemple musical 14 : Couple au repos, Michel Gonneville, mesures 1 à 8.

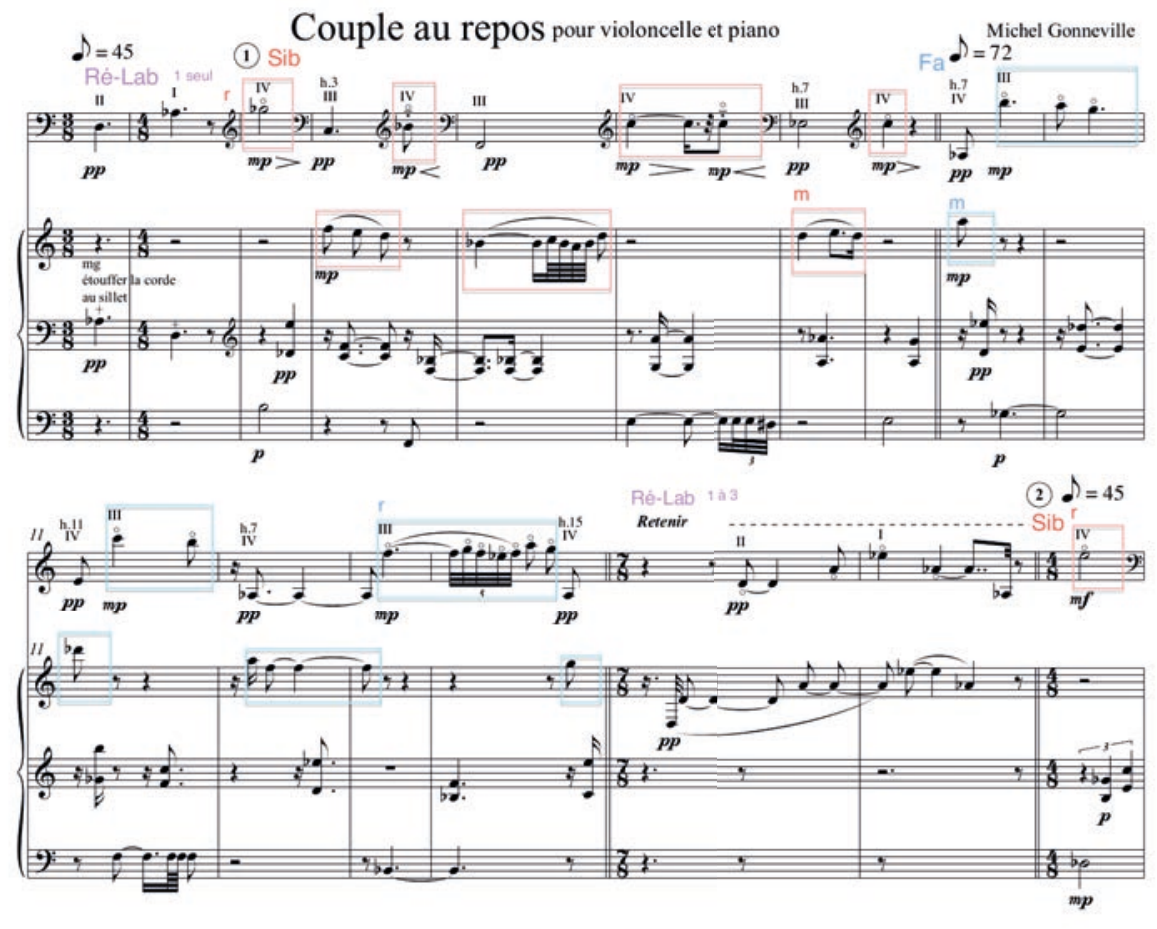

C Michel Gonneville 2011

Exemple musical 15 : Couple au repos, Michel Gonneville, mesures 1 à 17.

De plus, Gonneville "transpose " la mélodie tonale de Chopin sur le mode acoustique ayant le si bémol comme note "tonique ». La phrase suivante préserve chez Gonneville le profil mélodique original de la phrase de Chopin (transposé modalement de nouveau mais " en $f a$ ") mais en l'associant cette fois à une rotation de la phrase rythmique. 


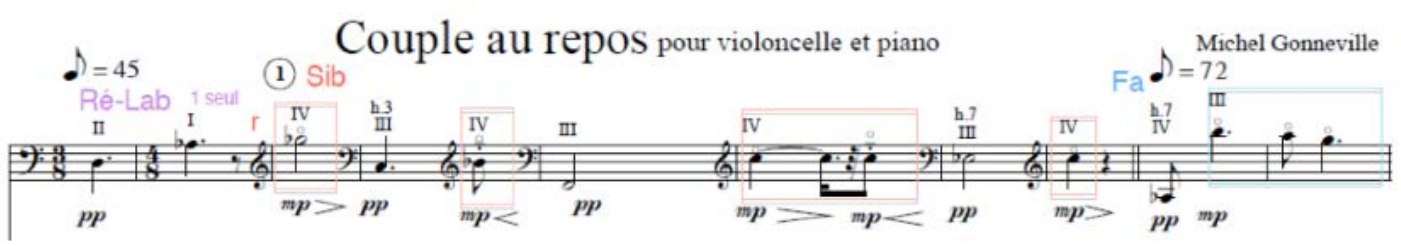

Exemple musical 16: Couple au repos, Michel Gonneville, pièce intégrale.

Browsing Agon (2008) : ou la "promenade dans un musée »

Michel Gonneville présente à l'interprète cette analyse en introduction de la partition : "L'œuvre est composée de dix miniatures rassemblées en un seul mouvement et reliées entre elles par un "refrain" ou une "ritournelle" appelé Browsing. " Elle s'appuie sur le ballet Agon de Stravinsky, chorégraphié par Georges Balanchine et créé en 1957.

Le tableau ci-dessous, élaboré par le compositeur, indique l'emplacement chronométré des emprunts dans chaque miniature :

\begin{tabular}{|c|c|}
\hline Browsing Agon (enregistrement Aventa) & $\begin{array}{l}\text { «Stravinsky Agon» (enregistrement Robert } \\
\text { Craft) }\end{array}$ \\
\hline « Krakatoa » 0:32 & $\begin{array}{l}\text { «Coda - Doppio Lento Quasi Stretto - } \\
\text { Coda } 1: 18\end{array}$ \\
\hline « Zéphyr, or Amnesia » 1:43 & «Prelude $» 0: 13$ \\
\hline «Bisbigliando » 2:40 & $\begin{array}{l}\text { «Second Pas de Trois : Bransle Simple» } \\
0 \cdot 00\end{array}$ \\
\hline «Sushi » 3:05 & «First Pas de Trois : Saraband-Step » 0:00 \\
\hline «Flamenco » 5:14 & «Bransle Gay » 0:00 \\
\hline «Newt » 0:00 & «Double Pas de Quatre » 0:00 et 0:43 \\
\hline « Hocketus with Klangfarben » 7:30 & «Coda » 0:00 \\
\hline $\begin{array}{l}\text { "Hot sandy beach, with sporadic } \\
\text { raindrops " 8:25 }\end{array}$ & $\begin{array}{l}\text { «Pas de Deux - Piu Mosso - L'istesso } \\
\text { Tempo - Refrain » 0:00 }\end{array}$ \\
\hline «Multiphonics » 11:33 & « Gaillarde » 0:00 \\
\hline $\begin{array}{l}\text { "Turkey - the country - Shoes and } \\
\text { Wine »13:13 }\end{array}$ & $\begin{array}{l}\text { «Pas de Deux - Piu Mosso - L'istesso } \\
\text { Tempo - Refrain » 3:12 }\end{array}$ \\
\hline «Agon (browsing back)» 14:24 & \\
\hline
\end{tabular}

Figure 2: Correspondances entre les dix miniatures Browsing Agon de M. Gonneville et des extraits d'Agon de Stravinsky

Chaque miniature porte le titre d'une "idée " proposée par un instrumentiste de l'ensemble Aventa et met à l'avant-plan l'instrument de la personne qui a proposé cette idée. Cet instrument principal peut être associé à d'autres instruments pour former des groupes comprenant de 1 à 10 instruments. Le nombre d'instruments par groupe suit cet ordre : 1928374655 et pour la conclusion 10 .

Chaque miniature est centrée sur une note principale. La succession des notes principales résulte d'une lecture des extrêmes au centre d'une série d'harmoniques naturels de do, ajustés au tempérament : 1-19-3-17-5-15-7-13-9-11, soit do-mib-sol-réb- 
mi-si-sib-lab-ré-fa\#. Ces notes principales servent de note initiale pour un mode, de note centrale, de tonique etc. Les deux notes manquant à la série de dix sons pour constituer le total chromatique : $f a$ - harmonique 21 de do - et $l a$ - harmonique 27 sont ajoutées pour constituer la série dodécaphonique du refrain pointilliste. Le $f a$ sert de plus comme note prépondérante dans la Coda (Agon) et le la pour l'introduction et la conclusion (Tuning). Dix des douze notes de la série sont accompagnées d'une note secondaire choisie pour constituer au total une série de dix intervalles différents. L'ordre de ces intervalles reproduit celui des intervalles entre une fondamentale $x$ et ses harmoniques impairs successifs (de 3 à 21), soit 7-4-10-2-6-8-11-1-3-5, présenté dans l'ordre des extrêmes au centre : 5-7-3-4-1-10-11-2-8-6.

Plusieurs miniatures utilisent un mode, certaines une série de douze sons. Il y a une recherche de variété dans les différents « modes » ou séries utilisés dans les différentes miniatures. Ce choix est influencé par la thématique des «idées " proposées. Quant aux modes, ce choix peut concerner le nombre de notes du mode ou sa structure, et l'intervalle de reproduction du mode (modulo).

\section{"Krakatoa "}

Nous nous attacherons plus particulièrement à la première miniature. "Krakatoa » se bâtit à partir de l'évocation de deux explosions volcaniques et des retombées de cendres et de pierres.

Le matériau issu du ballet Agon de Stravinsky provient plus précisément du Pas de 2, Stretto (mesures 512 à 519, partie du piano).

Exemple musical 17 : Agon, Stravinsky, mesures 512 à 519. 


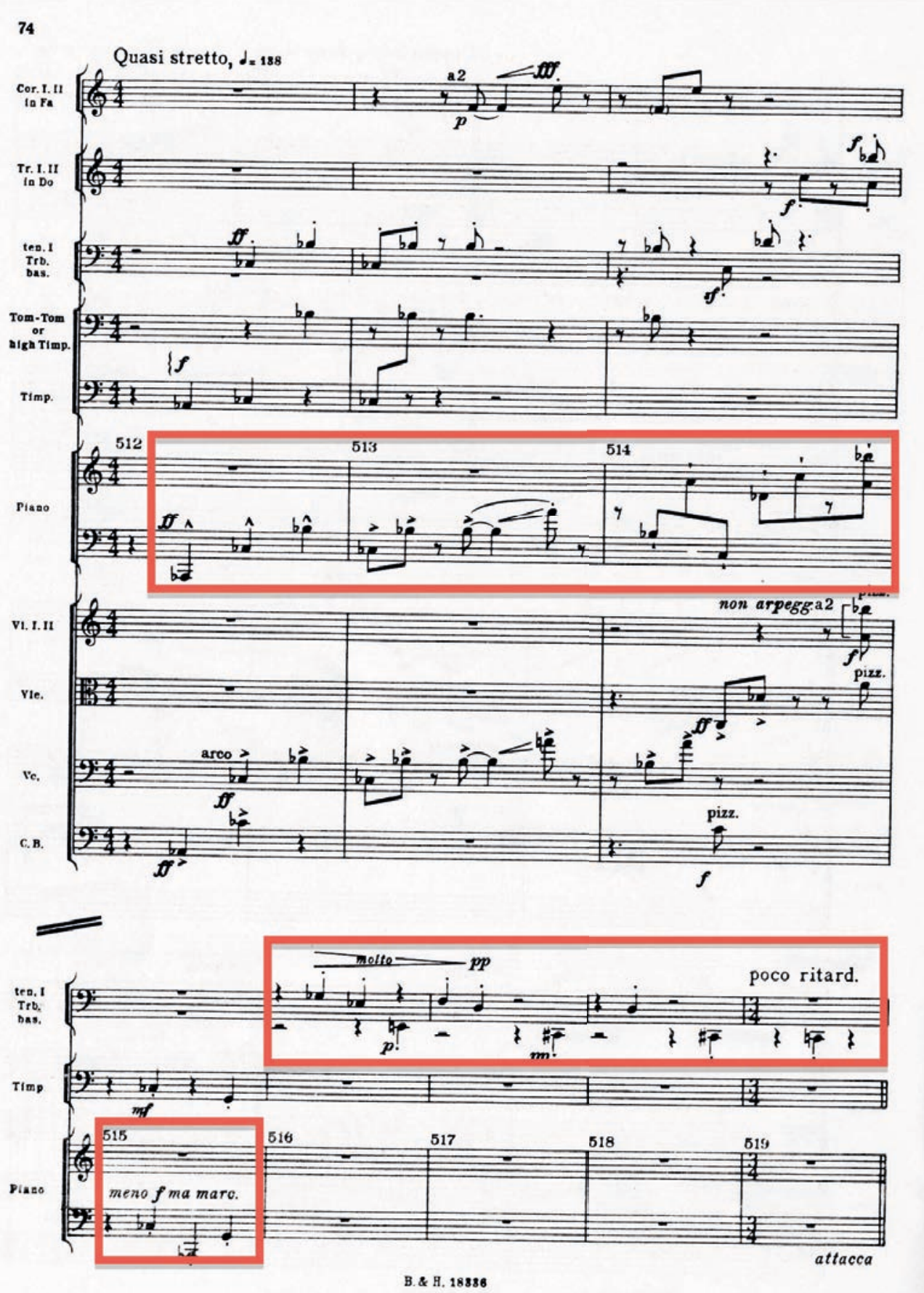

Exemple musical 18 : Partition de Agon, Stravinsky, "Pas de 2 ", Stretto, mesures 512 à 519 (partie du piano). 


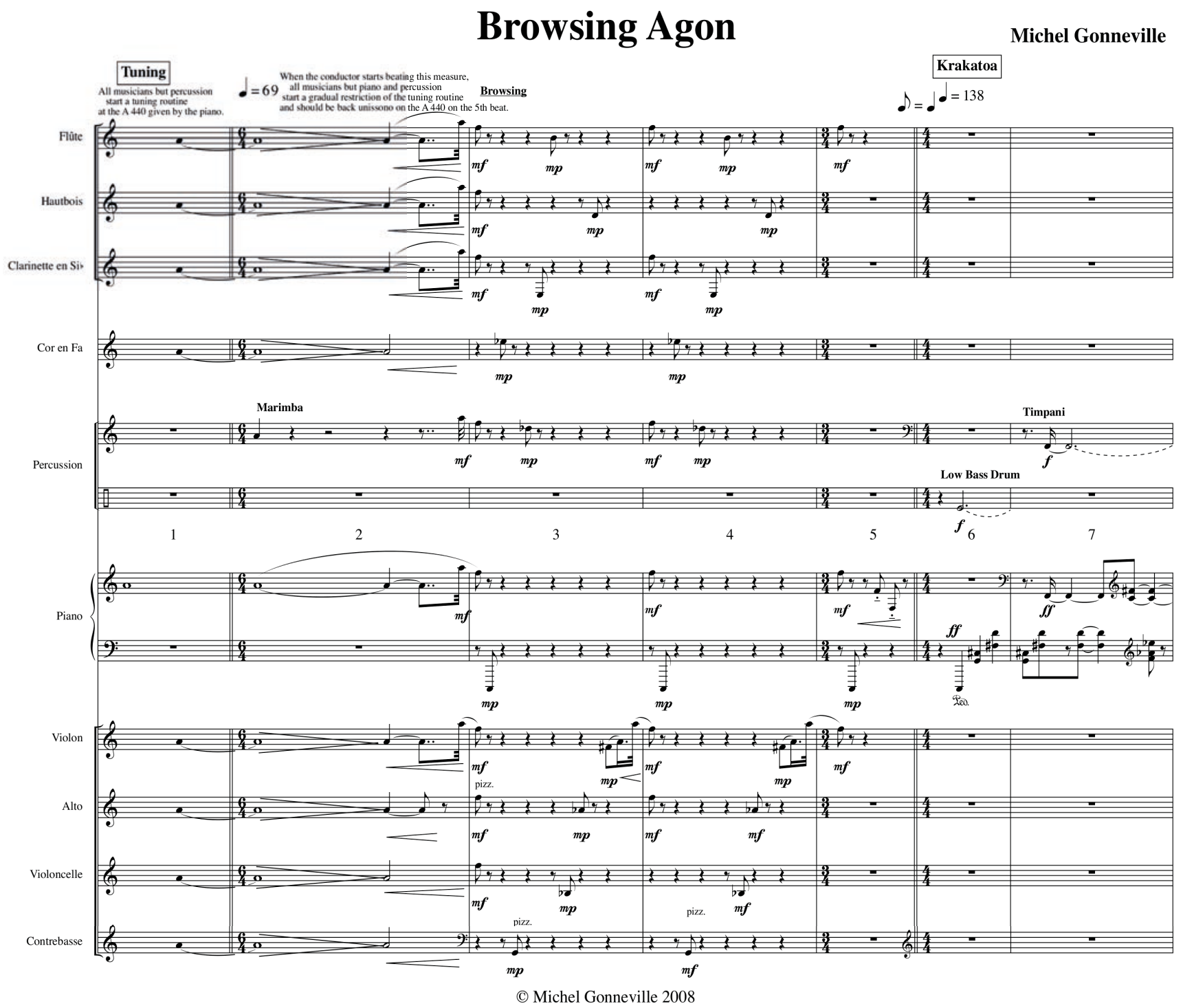

Exemple musical 19: Partition de "Krakatoa», Browsing Agon, Michel Gonneville, mesures 1 à 7.

À la mesure 6 débute un canon rythmique à deux voix à partir du rythme originel de Stravinsky, soit (en doubles croches) :

Dux 4- 4- 4- -2- 4- 6- 6- 2- 2- 2- 2- 4- 6- 4- 4- 8- 4- 4- 4- 4- 8- 8- 8- 8- 8

Comes 7- 7- 7- 5- 7- 9- 9- 5- 5- 5- 5- 7- 9- 7- 7- 11- 7- 7- 7- 7- 11- 11- 11- 11-11

La transposition du profil mélodique de l'exposition sérielle de Stravinsky est distribuée sur trois modules d'un mode non-octaviant (octave + quarte) de onze sons (harmoniques $8,9,10,11,12,13,14,15,17,19,21)[=0,2,4,6,7,8,10,11,13,15$, 17]. 

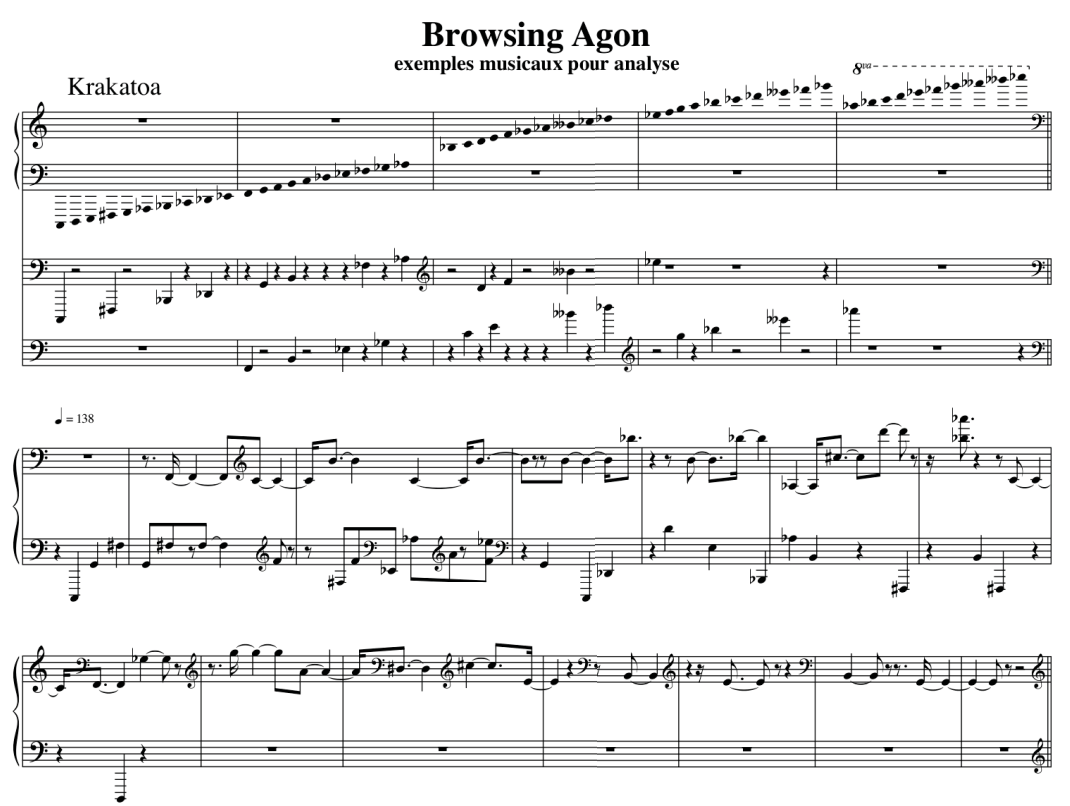

Exemple musical 20 : Mode non octaviant et canon-dux et comes.

Le canon à deux voix est à l'octave + quarte. La voix 1 est écrite sur le mode commençant sur le do grave, la voix 2 est écrite sur la transposition de ce même mode commençant sur le $f a$ une octave et une quarte plus haut (voir les $2^{e}$ et $3^{\text {e }}$ systèmes de l'exemple 20).

Pour les canons comme pour les désinences, Gonneville augmente la densité harmonique par une harmonisation parallèle sur le mode : densité d'un son sur les notes du premier module, densité de deux sons sur le deuxième (selon le modèle : harmoniques $2+3$ formant quinte), densité de trois sons sur le troisième (selon le modèle : harmoniques $4+5+7$, équivalent à un accord de $7^{\mathrm{e}}$ naturel sans quinte).

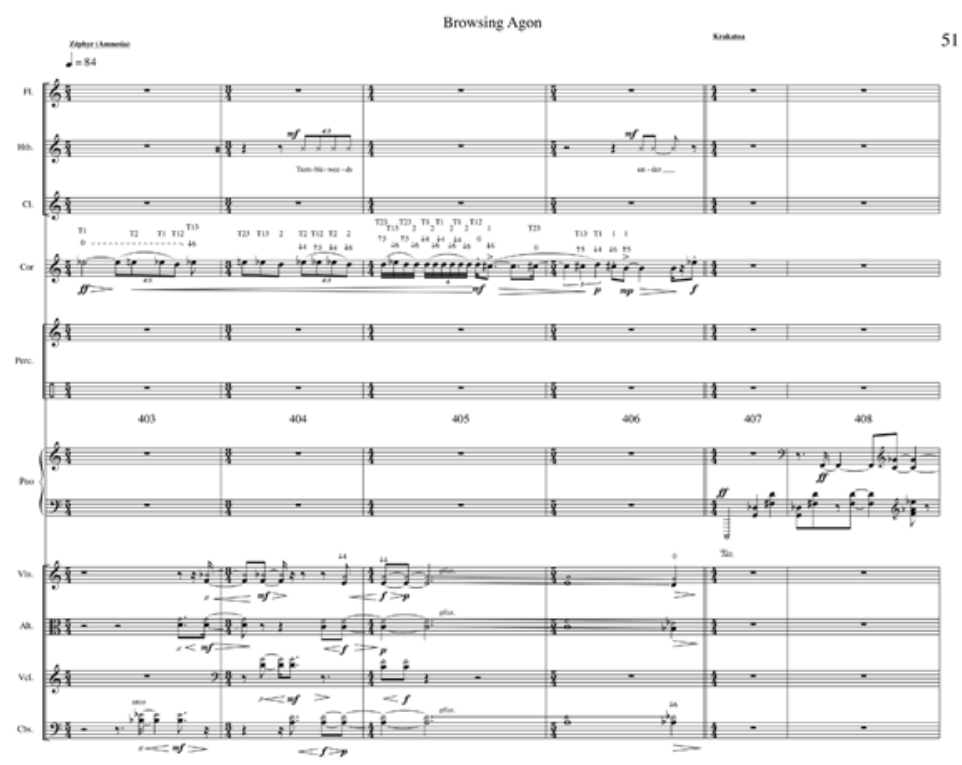

Exemple musical 21 : "Krakatoa", Browsing Agon, Michel Gonneville, mesures 8 à 14 - densités harmoniques (écoute de la pièce intégrale). 
On le voit, il y a à la fois présence structurelle en filigrane du passé et proclamation moderniste par l'utilisation d'un langage atonal et d'une complexité organisée et rationnalisée du matériau. On peut parler de strates historiques et de palimpseste, et selon la terminologie de Genette, d'hypertexte, c'est-à-dire d'une création littéraire ou artistique obtenues par dérivation d'un texte préexistant (Genette, 1982), pour caractériser l'esthétique de Michel Gonneville. Ce qui participe, en revanche, du postmodernisme chez ce compositeur est la quasi systématisation de ce procédé d'hypertextualité dans son œuvre, comme si la considération de l'histoire et son rôle dans le présent devenaient le sujet et l'enjeu principal de la composition d'une œuvre.

\section{CONCLUSION}

Si l'on reconsidère le tableau des différences d'attitudes entre le modernisme et le postmodernisme et si l'on met en évidence les termes correspondant aux démarches de Michel Gonneville et d'Ofer Pelz, on constate en vert pour les deux et, en rouge spécifiquement pour Michel Gonneville, et en gris clair spécifiquement pour Ofer Pelz, des caractéristiques appartenant aux deux courants (voir tableau en fin d'article). Ofer Pelz interprète et utilise la modernité du passé pour consacrer la sienne. Son modernisme repose sur son attachement viscéral à la cohérence formelle, à la complexité comme valeur, à l'unité et à la maîtrise du matériau, ainsi que sur son rejet de l'éclectisme. Le pont stylistico-esthétique qu'il jette entre deux époques éloignées de quatre siècles est construit à partir des notions de modernisme et d'avant-garde, notion apparue au début du $\mathrm{Xx}^{\mathrm{e}}$. Il associe les dissonances de son propre langage à celles pratiquées par Gesualdo.

Pour Michel Gonneville, il ne s'agit pas, en distordant et métamorphosant le matériau passé, de creuser la distance avec le passé, mais de rivaliser avec les " pères ", de continuer de tracer la transmission des découvertes de génie et valoriser l'héritage. Il cherche à perpétuer la tradition tout en racontant autre chose dans chaque œuvre. Issu d'une génération antérieure à Pelz, Michel Gonneville fait montre d'un retour au passé et d'un historicisme qui demeurent une réaction contre l'avant-gardisme des années 1950. Par conséquent, l'esthétique du palimpseste, de la référence, bien que sans déférence, le second degré par la distanciation critique, revêt une dimension postmoderniste. Il n'en reste pas moins qu'il se démarque d'un postmodernisme pur et reste ancré dans une pensée moderniste, par sa foi dans la ligne progressiste de l'esthétique et du langage et sa crainte de la régression, ainsi que son assimilation innovante utilisant les profils objectivés et conceptualisés de la tradition.

S'il est présomptueux de déclarer la fin d'un rapport postmoderne au passé, les deux cas étudiés ici, celui de Michel Gonneville et d'Ofer Pelz nous montrent que le modernisme demeure : ces deux compositeurs aux démarches d'emprunt clairement opposées sont préoccupés de nouveauté et de dialectique historique, de recherche de structuration formelle, de dépassement et de renouvellement du matériau historique. Ce qui importe est de trouver du nouveau, et, en tous les cas, d'aller au-delà de l'opposition antithétique entre le modernisme et du postmodernisme par l'innovation historique sans table rase.

À l'issue de cette étude, il semble ainsi possible d'appuyer les thèse de Heile du modernisme comme projet et celle d'Albrecht Wellmer selon laquelle la modernité et son 
point saillant qu'est le modernisme, est un « horizon indépassable ${ }^{15}$ », dans la mesure où même le postmodernisme n'est qu'un moment d'autocritique exacerbée qu'effectue la modernité devenue ironique et sceptique une fois privée de son « rêve de réconciliation », mais ce moment a préservé « l’esprit rationnel, subversif et expérimental de la démocratie, de l'art, de la science et de l’individualisme modernes, quoiqu'en disent les critiques postmodernistes ${ }^{16}$ ».

15 «La modernité est pour nous un horizon inatteignable dans un sens politique, cognitif, esthétique et moral "; notre traduction de cet extrait : "Modernity is for us an unsurpassable horizon in a cognitive, aesthetic and moral-political sense » (Wellmer 1991, p. vii).

16 "Sous son meilleur jour, le Postmodernisme peut être vu comme - une forme de modernisme autocritique - sceptique, ironique mais néanmoins implacable; un modernisme au-delà l'utopisme, scientifique et du fondationnalisme; bref, un modernisme postmétaphysique. [...] Une modernité postmétaphysique serait la modernité sans rêves de réconciliations ultimes, mais qui préserverait l'esprit rationnel, subversif et expérimental de la démocratie moderne, de l'art moderne, de la science moderne et de l'individualisme moderne $[. .]$.$» ; notre traduction de cet extrait : "[P]ostmodernism at its best might be seen as a self-cri-$ tical - a sceptical, ironic, but nevertheless unrelenting, -form of modernism; a modernism beyond utopianism, scientific and foundationalism; in short, a postmetaphysical modernism. [...] A postmetaphysical modernity would be modernity without the dream of ultimate reconciliations, but it would still preserve the rational, subversive and experimental spirit of modern democracy, modern art, modern science and modern individualism [...] » (ibid., p. vii-viii). 


\begin{tabular}{|c|c|}
\hline Modernisme & Postmodernisme \\
\hline Évolutionnisme & Fin de l'histoire \\
\hline Progressisme & $\begin{array}{l}\text { Dénégation du mythe du progrès et du } \\
\text { dépassement }\end{array}$ \\
\hline Complexité & Simplicité \\
\hline $\begin{array}{l}\text { Mouvement tourné vers l'avenir (Rea 1997, } \\
\text { p.61) }\end{array}$ & $\begin{array}{l}\text { Mouvement tourné vers le passé (Rea, loc. } \\
\text { cit.) }\end{array}$ \\
\hline $\begin{array}{l}\text { Culte de la nouveauté (« néopathie », Nattiez } \\
\text { 1993, p. 161), Dialectique de l'ancrage dans la } \\
\text { tradition versus radicalisme }\end{array}$ & $\begin{array}{l}\text { Fin des clivages nouveauté/ passé; } \\
\text { invention/académisme, rupture / } \\
\text { continuité entre révolution/réaction }\end{array}$ \\
\hline $\begin{array}{l}\text { Peut faire « table rase », avant-garde (ex. } \\
\text { ready-made) comme référence }\end{array}$ & $\begin{array}{l}\text { Sortie de l' « ère des ruptures » (Scarpetta } \\
1985, \text { p. } 28)\end{array}$ \\
\hline $\begin{array}{l}\text { Authenticité du discours, premier degré, référen- } \\
\text { cialité immédiate }\end{array}$ & $\begin{array}{l}\text { Toute l'œuvre est « métalangage », } \\
\text { "second degré » (Scarpetta 1985, p. 29). } \\
\text { « Mise en scène » de la « fascination » } \\
\text { à l'égard du passé (Ramaut-Chevassus } \\
\text { ca1998, p. 104). Le passé utilisé avec } \\
\text { «artifice » (Scarpetta, loc.cit.) }\end{array}$ \\
\hline Universalisme, autonomie de l'œuvre d'art & $\begin{array}{l}\text { Esthétique de l' «impureté » (Scarpetta } \\
\text { 1979). Acceptation de la diversité, de } \\
\text { la pluralité, des cultures multiples. La } \\
\text { musique n'est plus autonome, mais } \\
\text { soumise à des contextes sociaux, } \\
\text { politiques, culturels (Kramer 2002, p. 16) }\end{array}$ \\
\hline $\begin{array}{l}\text { Musique poḯtico-centrique (Nattiez 1993, } \\
\text { p. 180) }\end{array}$ & $\begin{array}{l}\text { Musique esthésico-centrique (Nattiez, loc. } \\
\text { cit.) }\end{array}$ \\
\hline Esthétique de la spécificité des codes & $\begin{array}{l}\text { Défie les barrières entre haut-style et } \\
\text { bas-styles, entre valeurs élitistes et valeurs } \\
\text { populaires (Kramer 2002, p. 16) }\end{array}$ \\
\hline $\begin{array}{l}\text { Culte de la forme, «synthèse ", "structure », } \\
\text { hiérarchisation, « design » formel (Hassan 1991, } \\
\text { p. 91) }\end{array}$ & $\begin{array}{l}\text { "Anarchie», « hasard» (Hassan 1991, } \\
\text { p. 91); Forme, processus }\end{array}$ \\
\hline $\begin{array}{l}\text { Transformation du matériau source, citations } \\
\text { exceptionnelles, dans un contexte sémantique } \\
\text { précis }\end{array}$ & Emprunt, citation, collage \\
\hline Unité, homogénéité & Éclectisme \\
\hline Extrapolation exclusive & Tri subjectif \\
\hline \multicolumn{2}{|l|}{ Assimilation innovante } \\
\hline Expression immédiate et intense & Expression \\
\hline \multicolumn{2}{|l|}{ Posture compositionnelle d'investigation } \\
\hline \multicolumn{2}{|l|}{$\begin{array}{l}\text { Idéaux de pureté et d'ascèse (silences, } \\
\text { fragments) }\end{array}$} \\
\hline $\begin{array}{l}\text { Diffusion dans le geste, l'énergie, la texture, } \\
\text { d'éléments passés }\end{array}$ & Passé réifié dans l'œuvre \\
\hline
\end{tabular}


Annexe : Moro Lasso, Ofer Pelz, partition annotée.

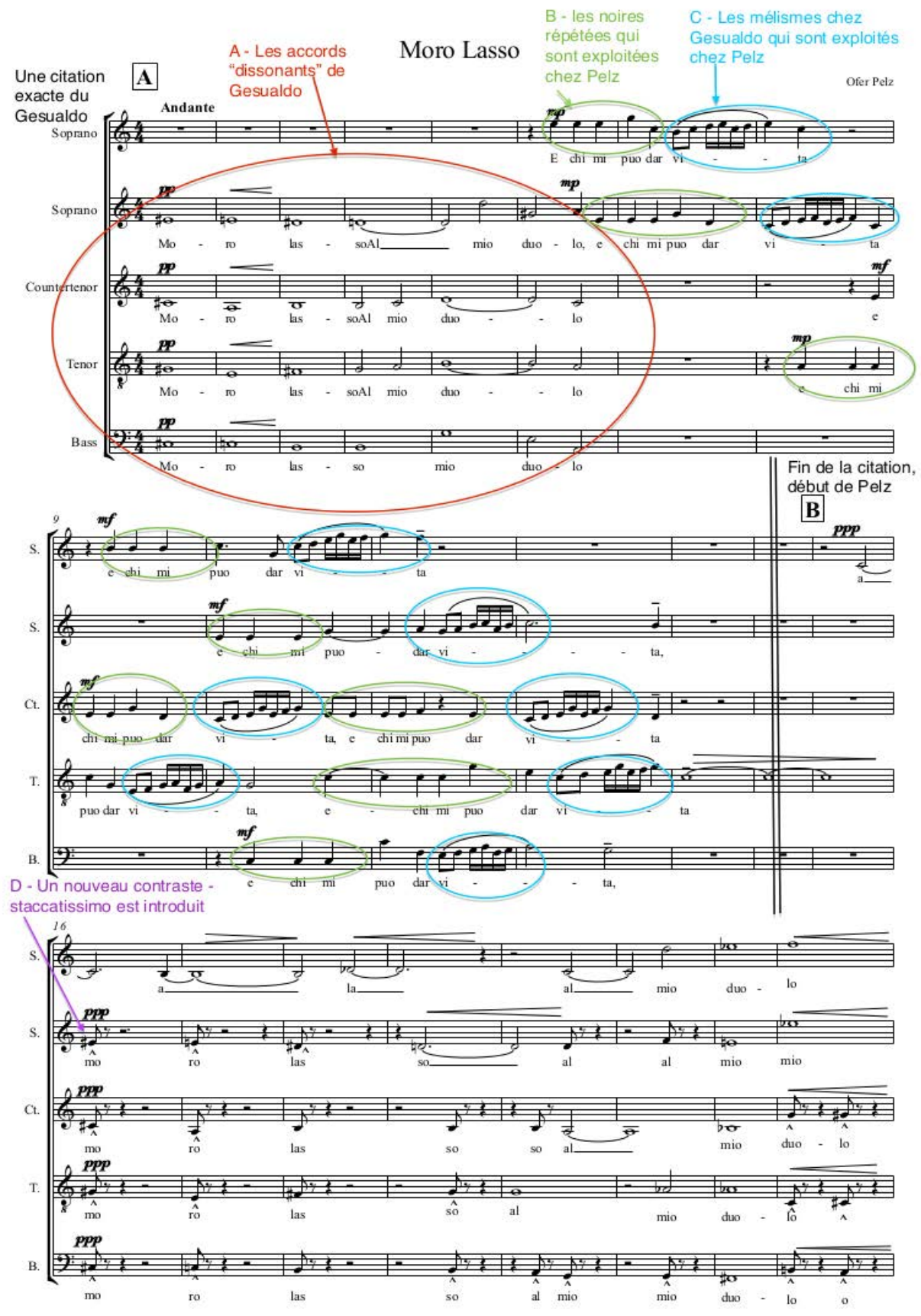


2

un développement des accords de Gesualdo
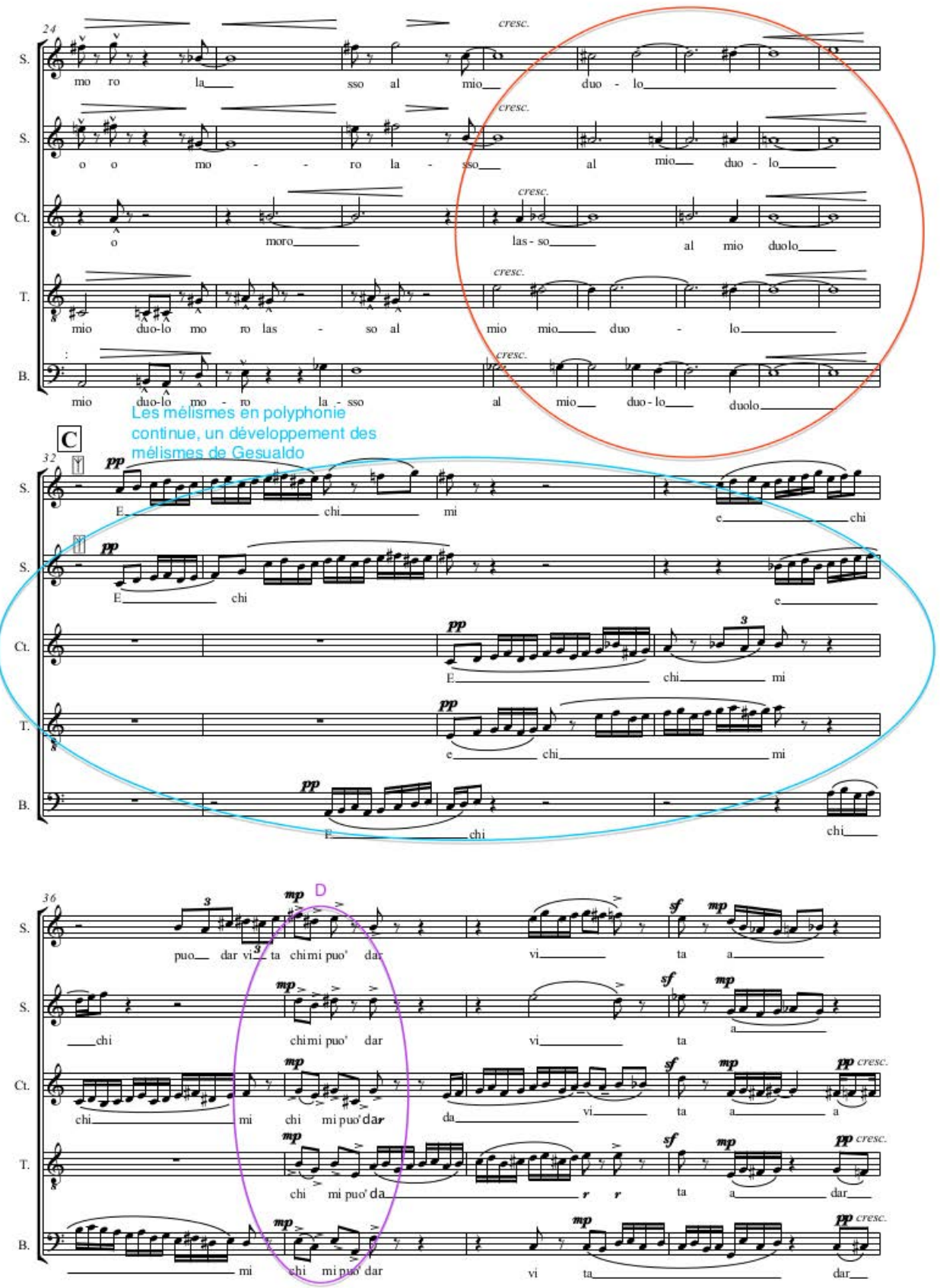

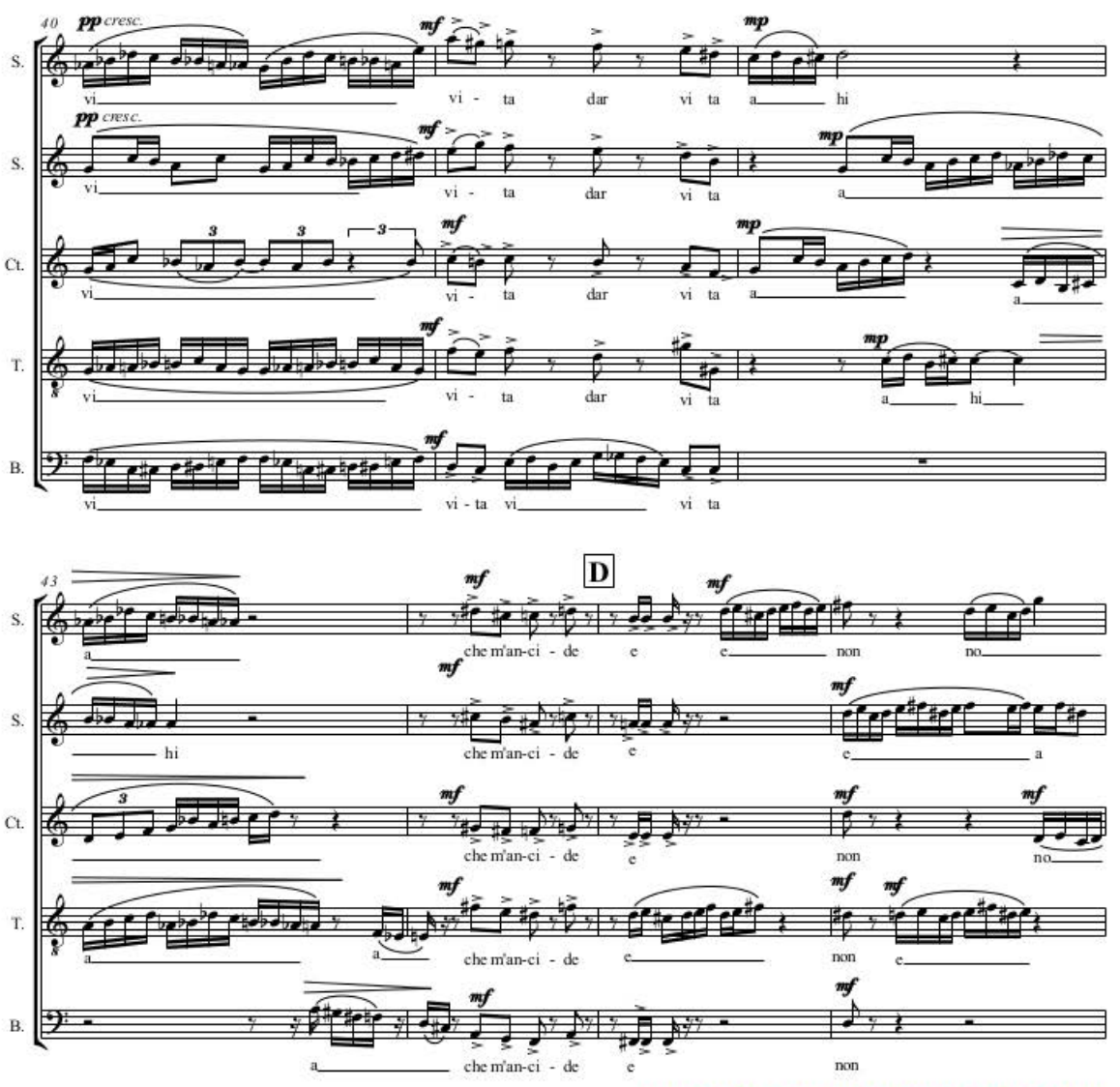

B est introduit, créant des harmonies plus claires.

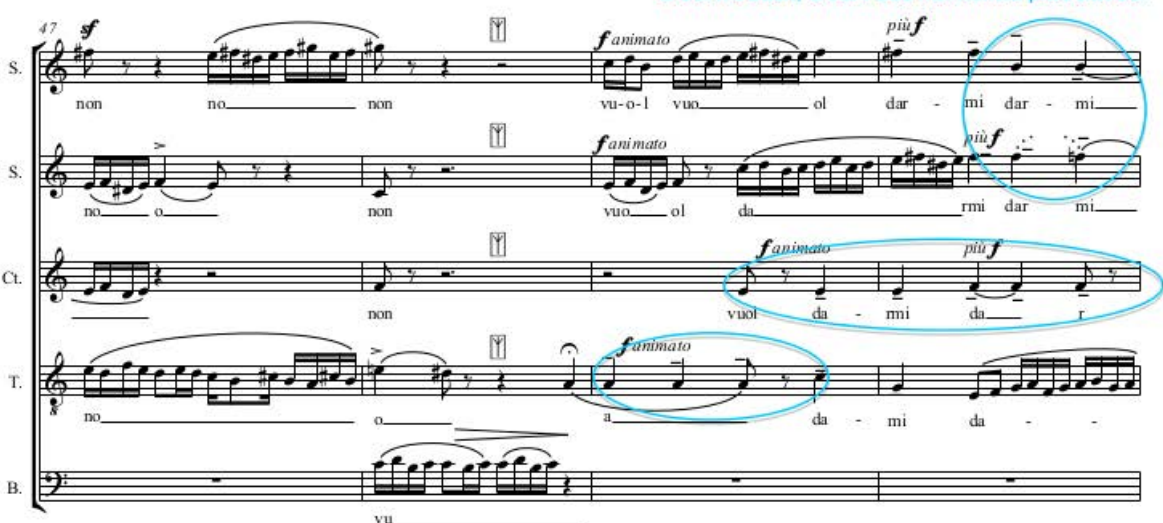



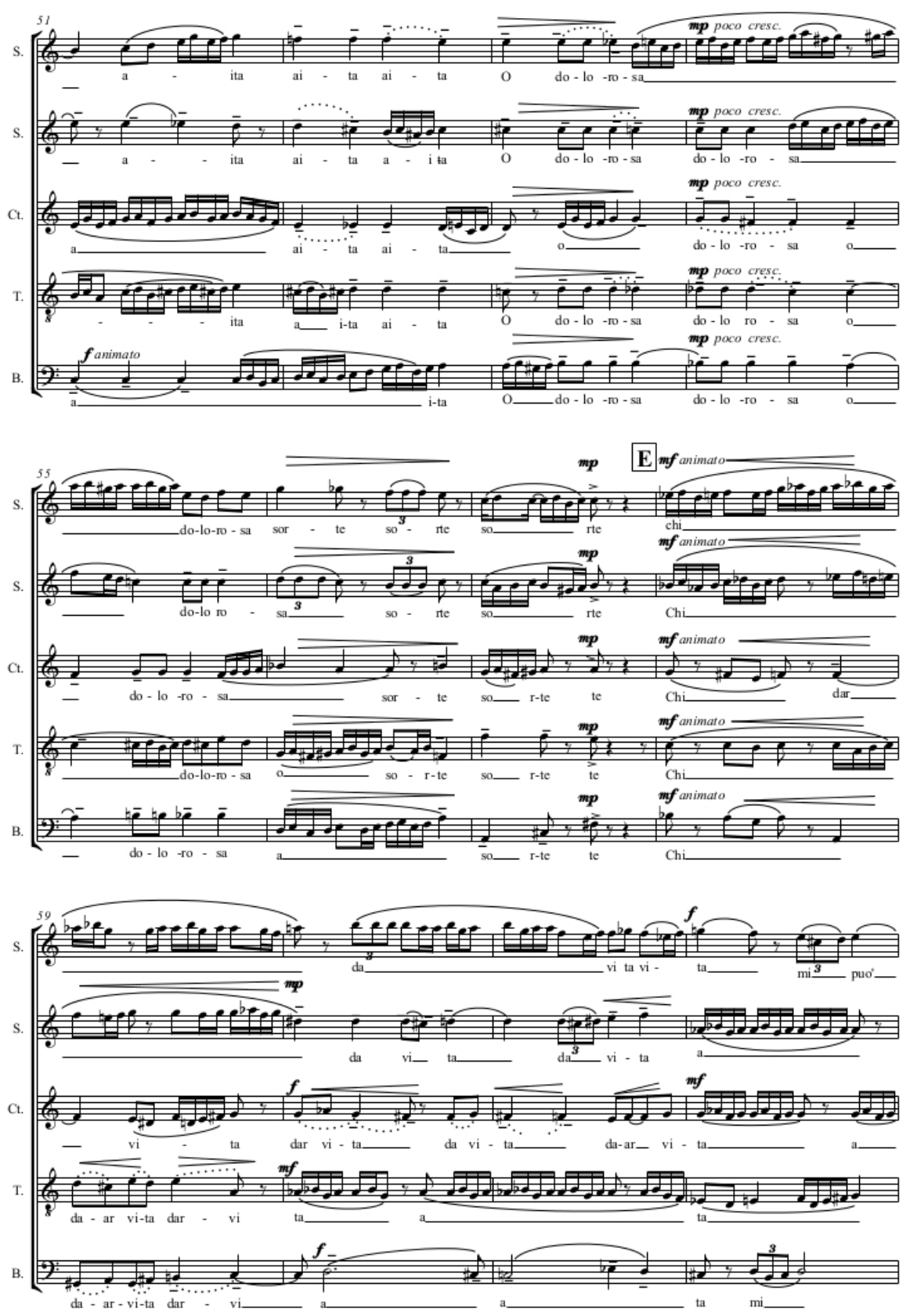

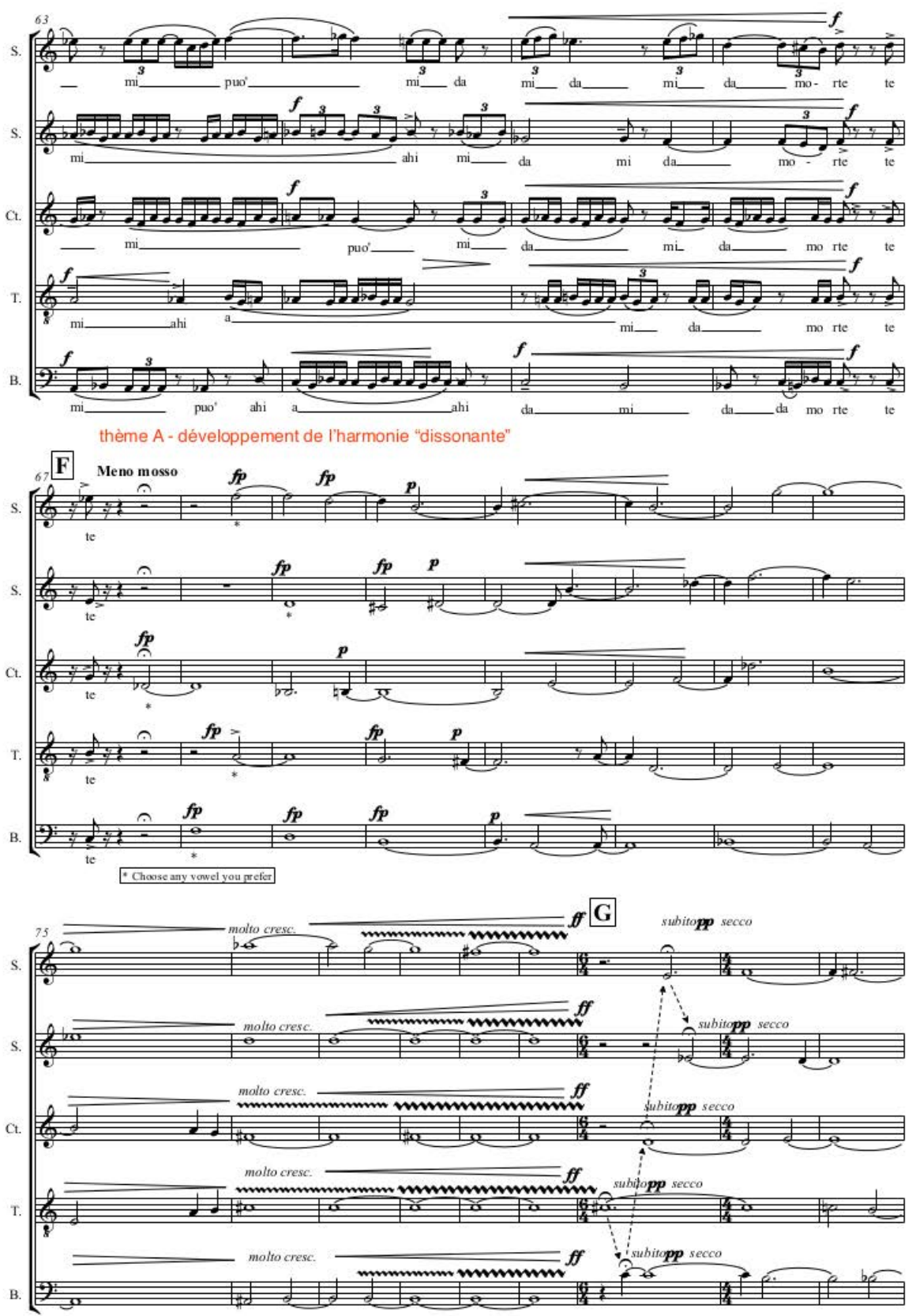


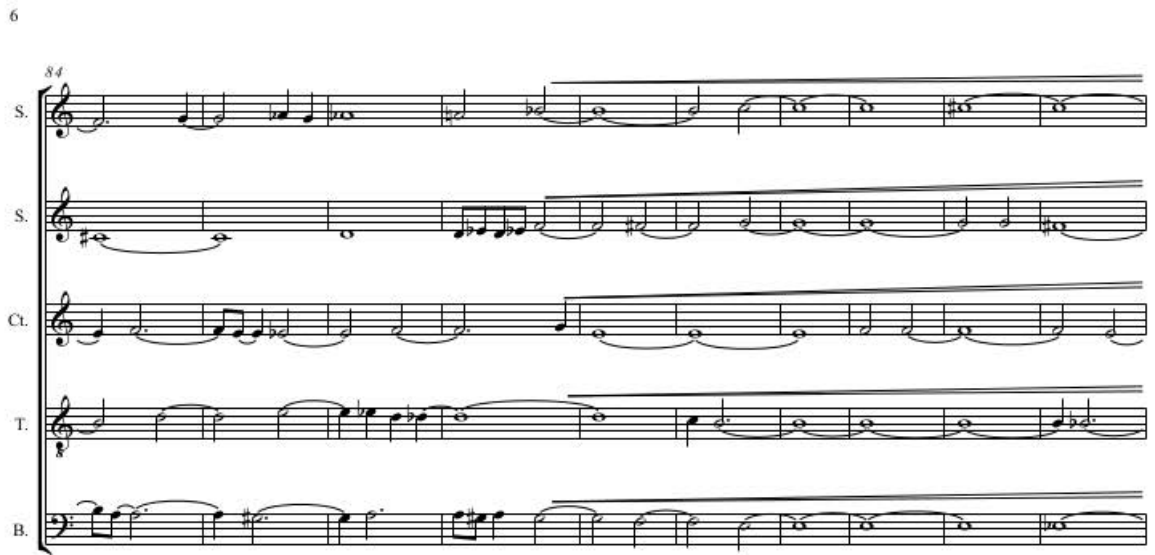

H $30 \mathrm{CA}$ nouveau matériau - (uniquement

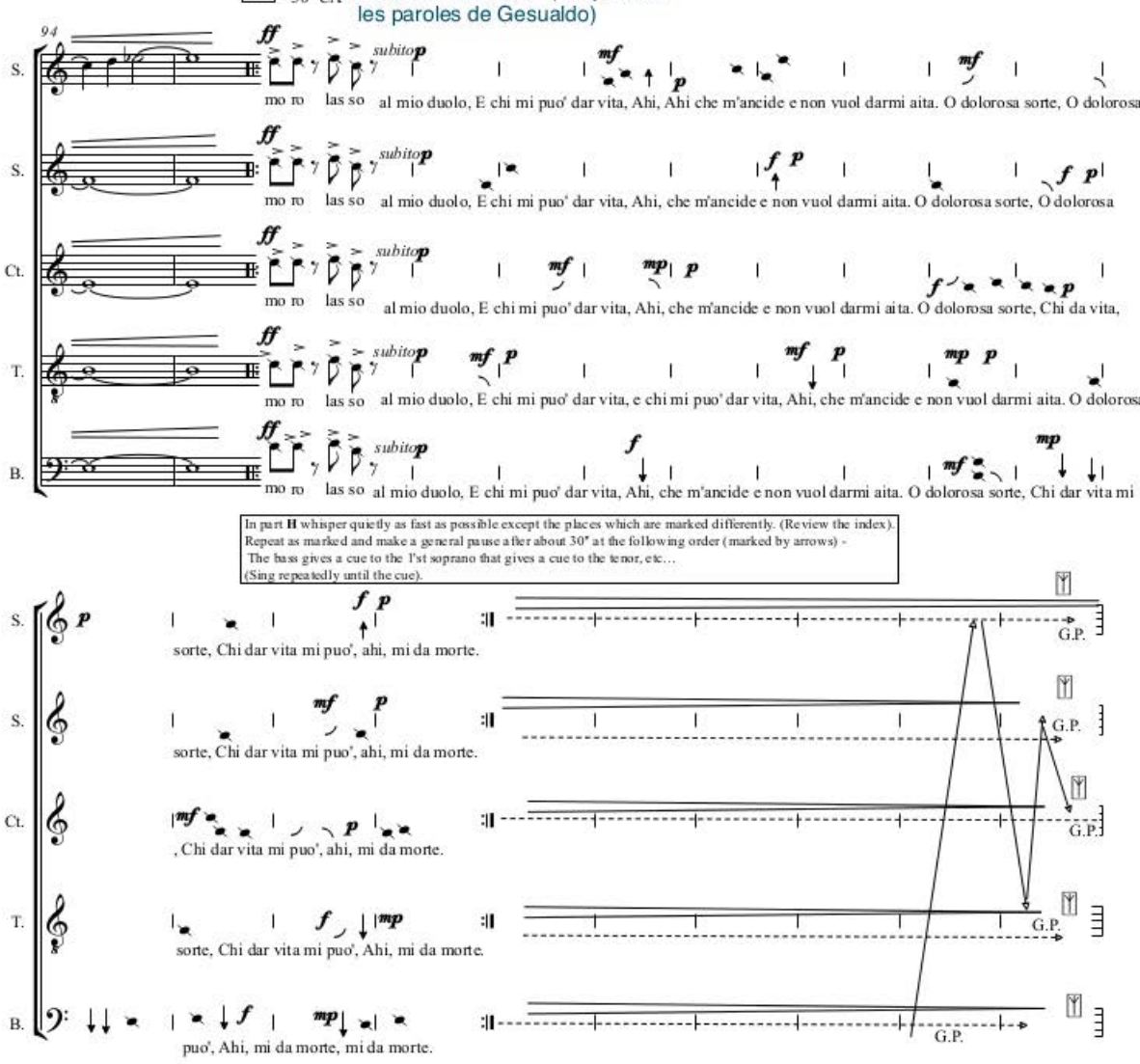



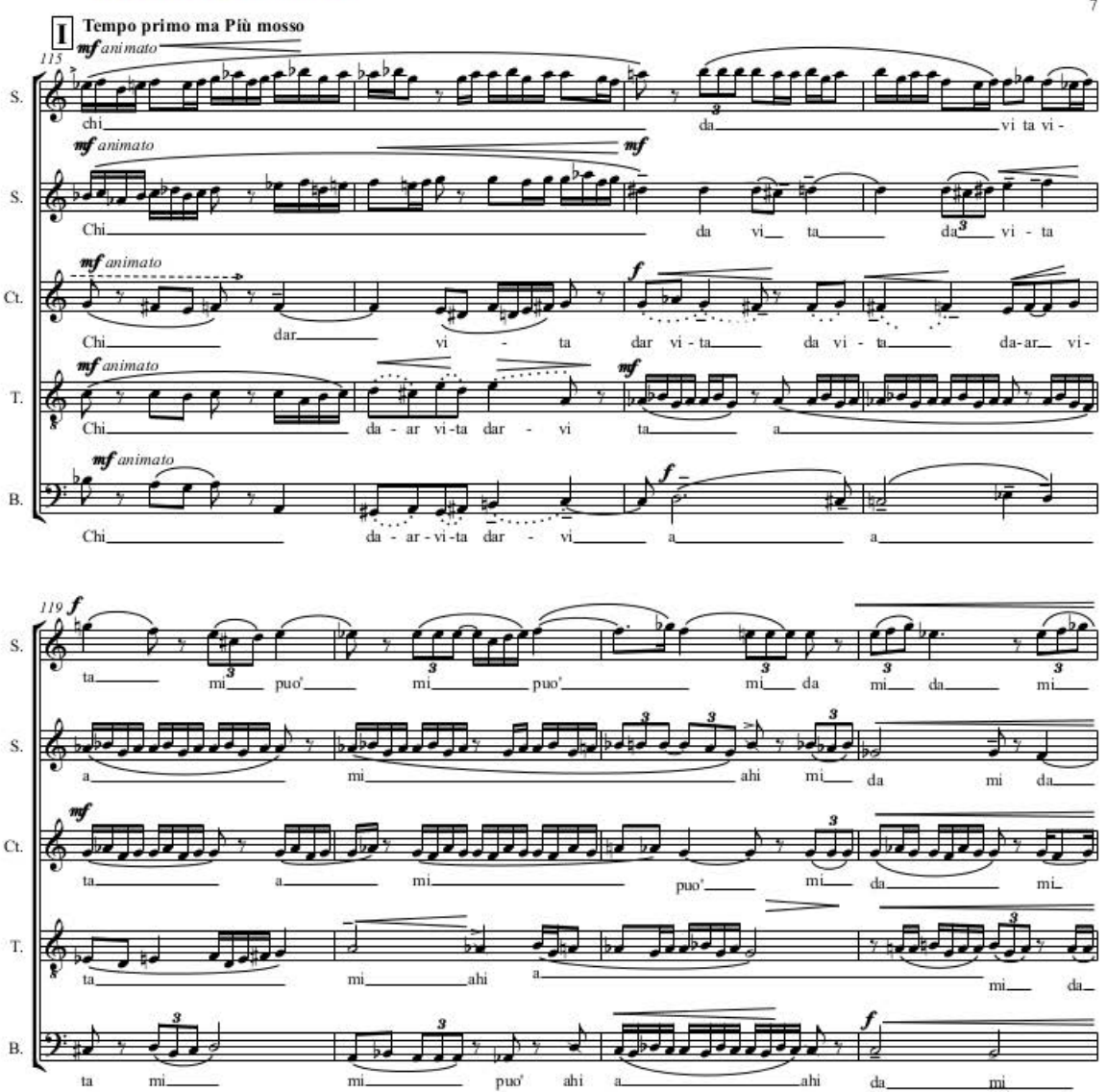

J Più mosso
ff cresc.

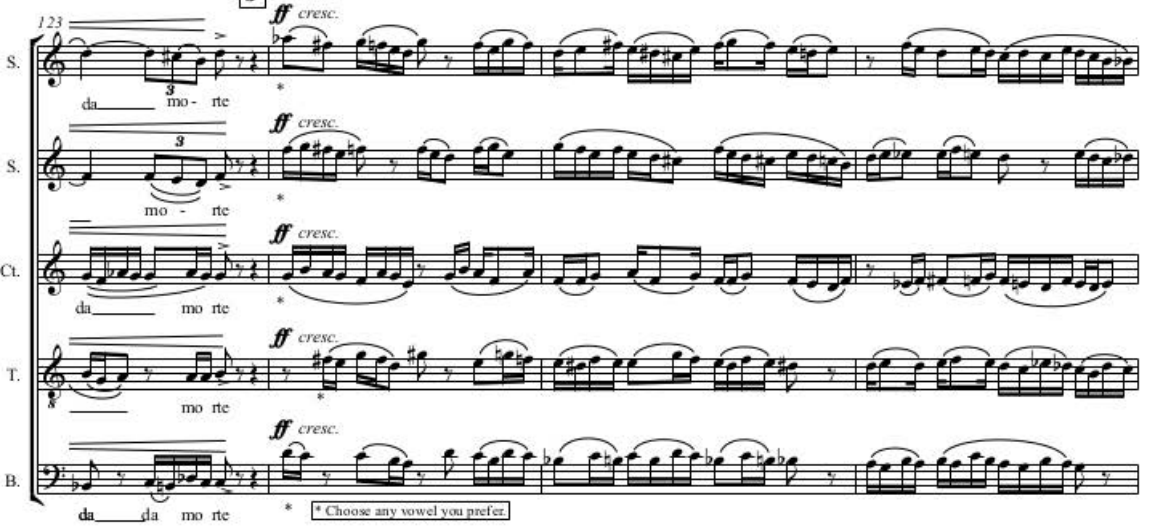




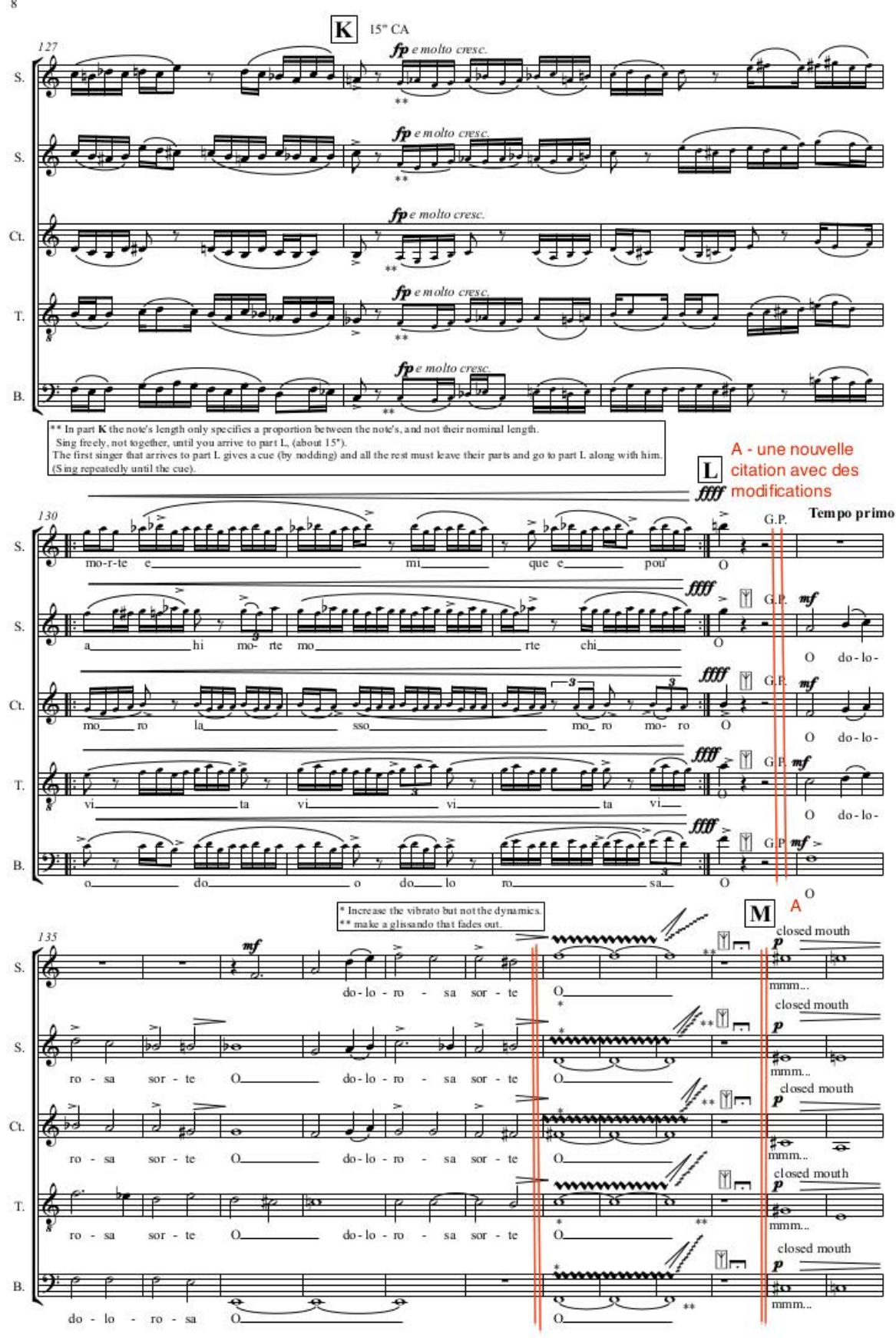



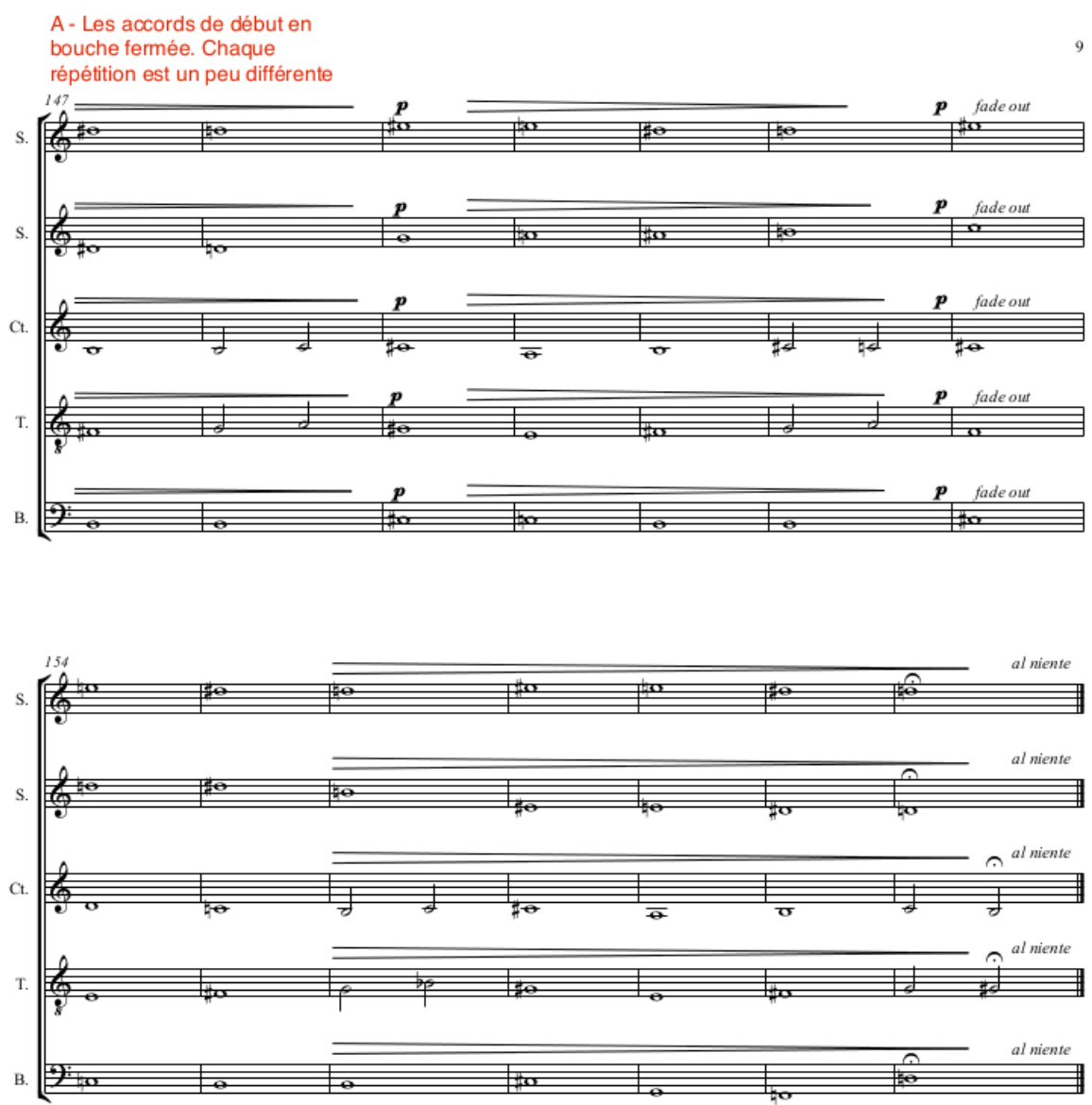


\section{SOURCES}

Réponses de Michel Gonneville et d'Ofer Pelz au questionnaire de l'auteur, juin 2012.

Entretiens de l'auteur avec Michel Gonneville, septembre 2012, octobre 2014.

Entretiens de l'auteur avec Ofer Pelz, juin 2012, septembre 2014.

\section{BIBLIOGRAPHIE}

Agamben, Giorgio (1999), The Man without Content, Stanford, Stanford University Press.

Berten, André (1991), « Modernité et postmodernité. Un enjeu politique? », Revue Philosophique de Louvain, vol. 89, n81, p. 84-112.

Burkholder, J. Peter (s.d.), " Borrowing ", Grove Music Online, Oxford University Press, www.oxfordmusiconline.com/subscriber/article/grove/music/52918, consulté le 6 mai 2015.

Croft, John (2009), «Fields of Rubble. On the Poetics of Music after the Postmodern », dans Heile 2009, p. 25-38.

Dell'Antonio, Andrew (s.d.), "Postmodernism », Grove Music Online, Oxford University Press, www.oxfordmusiconline.com/subscriber/article/grove/music/A2259137, consulté le 6 mai 2015.

Dhomont, Francis (1990), «Le postmodernisme en musique. Aventure néo-baroque ou Nouvelle aventure de la modernité ", Circuit. Musiques contemporaines, vol. 1, n 1, p. 27-48.

Donin, Nicolas, et Laurent Feneyrou (2013), Théories de la composition musicale au XX siècle, Lyon, Symétrie.

Genette, Gérard (1982), Palimpseste. La littérature au second degré, Paris, Seuil.

Gloag, Kenneth (2012), Postmodernism in Music, Cambridge, Cambridge University Press.

Goldman, Jonathan (dir.) (2014), La création musicale au Québec, Montréal, Presses de 1'Université de Montréal.

Gonneville, Michel (1990), «Humeurs postmodernes ", Circuit. Musiques contemporaines, vol. 1, nº 1, p. $49-62$.

Harvey, David (1990), The Condition of Postmodernity. An Enquiry into the Origins of Cultural Change, Cambridge, Wiley-Blackwell.

Hassan, Habib Ihab (1999), The Postmodern Turn. Essays in Postmodern Theory and Culture, Colombus, Ohio State University Press.

Heile, Björn (dir.) (2009), The Modernist Legacy. Essays on New Music, Farnham, Ashgate.

Ibedi Varga, Áron (1990), «Le récit postmoderne», Littérature, n77, p. 3-22.

Jameson, Fredric (1984), " Postmodernism, or the Cultural Logic of Late Capitalism ", New Left Review, vol. I, $\mathrm{n}^{\circ} 146$, p. 53-92.

Kabisch, Thomas (1995), «Zwischen Gesualdo und Reihentechnik. Alte Musik im Kontext der Poetik des späten Strawinsky ", Alte Musik im 20. Jahrhundert. Wandlungen und Formen ihrer Rezeption, New York, Schott, p. 113-130.

Kandinsky, Vassili ([1911]1989), Du spirituel dans l'art et dans la peinture en particulier, Paris, Denoël.

Kramer, Jonathan D. (2002), "The Nature and the Origins of musical Postmodernism ", dans Lochhead et Auner 2002, p. 72-89.

Lochhead, Judy, et Joseph Auner (dir.) (2002), Postmodern Music/Postmodern Thought, New York, London, Routledge.

Lyotard, Jean-François (1979), La condition postmoderne. Rapport sur le savoir, Paris, Minuit. 
Lyotard, Jean-François (1986), Le postmodernisme expliqué aux enfants. Correspondance 1982-1983, Paris, Galilée.

Metzer, David (2003), Quotation and Cultural Meaning in Twentieth-Century Music, Cambridge, Cambridge University Press.

Metzer, David (2009), Musical Modernism at the Turn of the Twenty-First Century, Cambridge, Cambridge University Press.

Molino, Jean (1997), «Classiques et classicisme à l'âge postmoderne », Circuit. Musiques contemporaines, vol. $1, \mathrm{n}^{\circ} 1$, p. $75-82$.

Nattiez, Jean-Jacques (1993), Le combat de Chronos et d'Orphée, Paris, Bourgeois.

Perloff, Marjorie (2002), 21st-Century Modernism. The New Poetics, Oxford, Blackwell.

Prévost, Hélène (1997) «Qu'est-ce que le postmodernisme musical ? ", Circuit. Musiques contemporaines, vol. 1, no 1, p. 9-26.

Ramaut-Chevassus, Béatrice (1998), Musique et postmodernité, Paris, Presses universitaires de France.

Rea, John (1990), « Postmodernité, que me veux-tu? », Circuit. Musiques contemporaines, vol. 8, nº 1 , p. $55-70$.

Scarpetta, Guy (1985), L'impureté, Paris, Grasset.

Timms, Andrew (2009), « Modernism's Moment of Plenitude », dans Heile 2009, p. 13-24.

Toscano, Maria Manuela (1999), "Chemins vers une esthétique de l'inquiétude dans la musique de Gesualdo ", International Review of the Aesthetics and Sociology of Music, vol. 30, n 1, p. 27-53.

Wellmer, Albrecht (1991), The Persistence of Modernity. Essays on Aesthetics, Ethics and Modernism, Cambridge, MIT Press. 